\title{
Video Article \\ RGB and Spectral Root Imaging for Plant Phenotyping and Physiological Research: Experimental Setup and Imaging Protocols
}

\author{
Gernot Bodner ${ }^{1}$, Mouhannad Alsalem ${ }^{1}$, Alireza Nakhforoosh ${ }^{1}$, Thomas Arnold ${ }^{2}$, Daniel Leitner ${ }^{3,4}$ \\ ${ }^{1}$ Division of Agronomy, Department of Crop Sciences, University of Natural Resources and Life Sciences \\ ${ }^{2}$ Carinthian Tech Research AG, High Tech Campus Villach \\ ${ }^{3}$ Computational Science Center, University of Vienna \\ ${ }^{4}$ Simulationswerkstatt
}

Correspondence to: Gernot Bodner at gernot.bodner@boku.ac.at

URL: https://www.jove.com/video/56251

DOI: doi:10.3791/56251

Keywords: Environmental Sciences, Issue 126, Root System, Hyperspectral Imaging, RGB Images, Plant stress, Phenotyping, Rhizobox, Spectral Analysis

Date Published: 8/8/2017

Citation: Bodner, G., Alsalem, M., Nakhforoosh, A., Arnold, T., Leitner, D. RGB and Spectral Root Imaging for Plant Phenotyping and Physiological Research: Experimental Setup and Imaging Protocols. J. Vis. Exp. (126), e56251, doi:10.3791/56251 (2017).

\section{Abstract}

Better understanding of plant root dynamics is essential to improve resource use efficiency of agricultural systems and increase the resistance of crop cultivars against environmental stresses. An experimental protocol is presented for RGB and hyperspectral imaging of root systems. The approach uses rhizoboxes where plants grow in natural soil over a longer time to observe fully developed root systems. Experimental settings are exemplified for assessing rhizobox plants under water stress and studying the role of roots. An RGB imaging setup is described for cheap and quick quantification of root development over time. Hyperspectral imaging improves root segmentation from the soil background compared to RGB color based thresholding. The particular strength of hyperspectral imaging is the acquisition of chemometric information on the root-soil system for functional understanding. This is demonstrated with high resolution water content mapping. Spectral imaging however is more complex in image acquisition, processing and analysis compared to the RGB approach. A combination of both methods can optimize a comprehensive assessment of the root system. Application examples integrating root and aboveground traits are given for the context of plant phenotyping and plant physiological research. Further improvement of root imaging can be obtained by optimizing RGB image quality with better illumination using different light sources and by extension of image analysis methods to infer on root zone properties from spectral data.

\section{Video Link}

The video component of this article can be found at https://www.jove.com/video/56251/

\section{Introduction}

Roots provide several essential functions for plants such as storage of assimilates, anchorage of terrestrial plants in soil, and uptake and transport of water and nutrients ${ }^{1}$. From an evolutionary point of view, the formation of root axes is considered a fundamental precondition for the origin of land plants ${ }^{2}$. In spite of this important role, historically roots have occupied only a marginal position in biological research. In more recent times, however, there is increasing scientific interest in plant root systems as evidenced in Figure 1. 


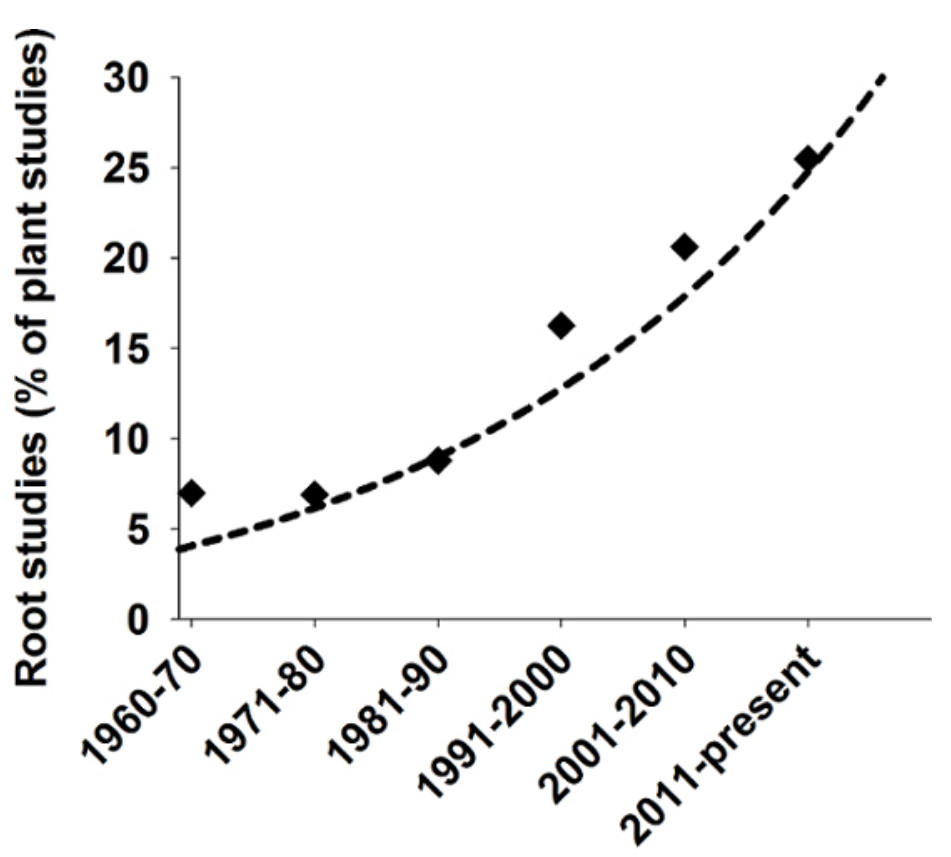

\section{Decade}

Figure 1: Relevance of root studies in plant sciences.

Number of root related studies as a percentage of all published plant studies in SCl journals over the last decades. Search result from Scopus using keywords "plant" and "plant AND root". Please click here to view a larger version of this figure.

Two main reasons can be hypothesized to underlie the recent advances in root research. First, terrestrial vegetation is exposed to more frequent environmental stresses as a result of global change ${ }^{3}$. In the context of agricultural crop production it is estimated that globally around $30 \%$ of the agricultural area are limited by water and phosphorus ${ }^{4,5}$. Stress reduction of crop yields are a main reason for significant yield gaps that are globally estimated at lower $50 \%$ of potential productivity for rainfed agro-ecosystems ${ }^{6}$. Besides low resource availability, this is also related to poor resource use efficiency, i.e. insufficient capacity of a plant to exploit available resources ${ }^{7}$. This results in losses of mobile resources such as nitrate which can negatively affect other ecosystems. The current global nitrogen use efficiency for example is estimated at $47 \%{ }^{8}$. Better resource use efficiency via improved management methods and cultivars is therefore of high importance for both sustained growth of agricultural outputs as well as for environmental sustainability. In this context plant roots are considered to be a key target for improved crops and cropping systems ${ }^{9,10}$.

A second important background for the recent interest in plant roots is technological advance in measurement methods. Root methods have long been restricted by two key challenges: for measurement of roots from plants growing in soil they had to be isolated for quantification, mostly by washing ${ }^{11}$, thereby disturbing the architectural arrangement of root axes. In-situ root observation using excavation methods, thereby conserving the natural location of roots in soil, have been used for botanical description ${ }^{12}$. Still they are very time-consuming and thus do not meet the throughput requirements of comparative structural-functional root system analysis. On the other hand high-throughput methods for root architecture measurement were mostly done on artificial media and for seedling plants ${ }^{13}$ where the extrapolation to the natural growth environment of plants is questionable ${ }^{14}$.

The recent boom of root research is tightly linked to the advance in imaging methods ${ }^{15}$. Imaging approaches in root studies can be roughly grouped into three types. First there are high resolution $3 \mathrm{D}$ methods such as CT and $\mathrm{MRI}^{16}$. These methods are most suitable to study interaction processes of plant roots with soil, such as drought induced xylem embolism ${ }^{17}$. Typically they are applied to comparatively small samples where they allow detailed observations. A comparison of CT and MRI for differently sized pots and fine root imaging is provided in ${ }^{18}$. Second, there are high-throughput imaging methods ${ }^{19,20}$. These methods are mostly based on common 2D RGB imaging of roots growing on artificial media (gel, germination paper) where high contrast allows comparatively simple dissection between roots and background. They are appropriate for high throughput comparison among seedling root traits of different crop genotypes under standardized artificial growing conditions $^{13}$. In between these two approaches are rhizobox methods: they use 2D imaging of roots growing in soil over longer time period and have medium throughput ${ }^{21,22}$. A recent challenge to (2D) root imaging is to capture also indicators of root functionality in addition to description of structure $^{23}$.

In the present paper we present the experimental protocols for imaging rhizobox grown root systems using (i) a cheap and simple custom-made RGB imaging setup and (ii) a more complex NIR imaging setup. Example results obtained from these two setups are shown and discussed in the context of plant phenotyping and plant physiological research. 
Protocol

\section{Rhizoboxes for plant growth}

NOTE: The experimental system uses rhizoboxes to grow plants for root imaging. First the design of the boxes and the substrate used are described, and then details on the filling procedure are given.

\section{Design of rhizoboxes}

1. Create rhizoboxes (Figure 2) with a back plate and side frames made of grey PVC with $15 \mathrm{~mm}$ strength. Use a box size of $300 \mathrm{~mm} x$ $1000 \mathrm{~mm}$. For the front window, use $6 \mathrm{~mm}$ mineral glass which is attached to the PVC frame by metal rails being screwed into the side walls.

2. Create three holes on the bottom frame to allow drainage of excess water. These holes can be optionally closed by plastic screws.

3. Before filling, adapt the inner diameter of the rhizobox (between $10 \mathrm{~mm}$ and $30 \mathrm{~mm}$ ) by inserting PC multiwall sheets. An inner space of $10 \mathrm{~mm}$ is recommended for most crops to reduce the weight (rhizobox weight without soil is $13.2 \mathrm{~kg}$ ) of the entire system.
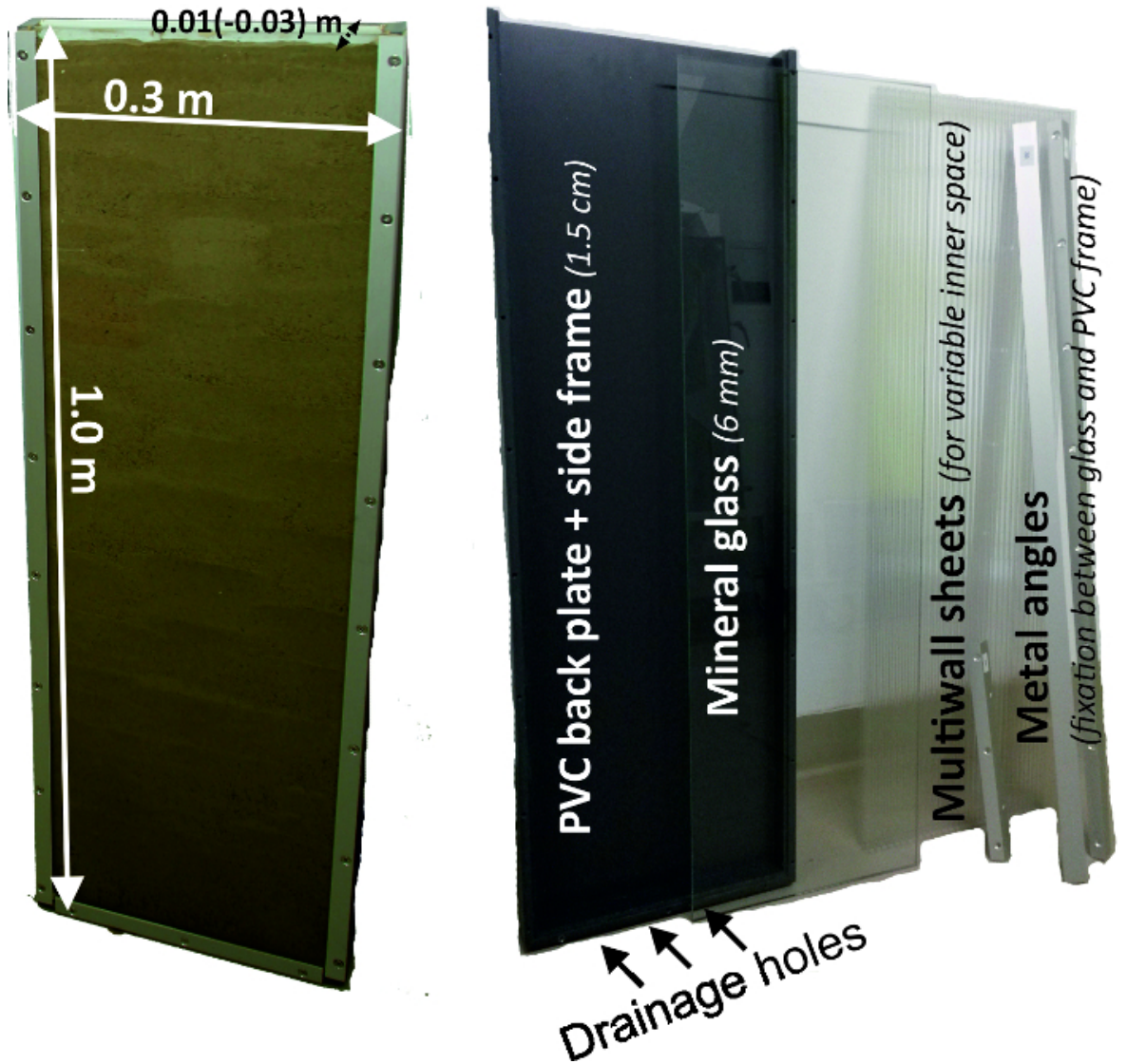

Figure 2: Rhizobox experimental system and its components.

Left figure shows the dimensions of a rhizobox and right figure its single components, being a grey PVC back plate with side frame, a front mineral glass, multiwall sheets for variable inner diameter and metal angles to fix the front glass to the back compartment. Please click here to view a larger version of this figure.

\section{Substrate}

1. Fill the rhizoboxes with field soil (for this experiment: silt loam top soil from a calcareous chernozem) sieved to $2 \mathrm{~mm}$ particle size.

2. Open the rhizoboxes for filling the substrate into the inner compartment (back plate with side frames) in horizontal position using prewetted substrate. Fill horizontally to avoid layering and segregation between fine and coarse particles which occurs when filling in vertical position by pouring the substrate through the upper opening.

3. Pre-wet the substrate before filling. Depending on the type of substrate (particularly on its silt and clay content), do not exceed a water content of $0.12-0.18 \mathrm{~cm}^{3 *} \mathrm{~cm}^{-3}$ to avoid smearing and structure degradation. Add the difference between pre-mixing and target water content after filling the substrate into the rhizoboxes.

NOTE: Filling with (oven-)dry substrate and subsequent addition of the entire water is not recommended as is can result in strong settlement of the substrate and formation of large cracks.

\section{Step by step filling example}


1. Define the target water content. Here it is initially set at $80 \%$ plant available water (PAW) where plants do not suffer from any water shortage.

2. Determine field capacity (FC) and permanent wilting point (PWP) of the substrate. Here obtain FC using a PVC tube of equivalent height $(100 \mathrm{~cm})$ as the rhizoboxes.

1. Close the tube at the bottom with a stubble having small drainage holes, add $1 \mathrm{~cm}$ of gravel to avoid closure of the holes from finer substrate and fill the substrate to the same bulk density as used for the rhizoboxes $\left(1.3 \mathrm{~g} \mathrm{~cm}^{-3}\right)$.

2. Saturate the tube with water until drainage occurs and leave it for two days to equilibrate (the resulting water content is per definition equivalent to field capacity), while covering the upper opening with cling-film to avoid evaporation. The field capacity value achieved for the soil in this experiment was $0.357 \mathrm{~cm}^{3} \mathrm{~cm}^{-3}$.

NOTE: Water content at PWP has to be known in advance from standard soil physical methods (e.g. pressure plate measurements ${ }^{24}$ ) or from texture based pedotranfer functions ${ }^{25}$. Here it is equal $0.12 \mathrm{~cm}^{3} \mathrm{~cm}^{-3}$ for the soil used.

3. When measuring $\mathrm{FC}$ by pressure plate extraction too, take the water content at a matrix potential of $h=-100 \mathrm{hPa}$ and not $\mathrm{h}=-330$ $\mathrm{hPa}$ in order to correspond to the rhizobox geometry (height of $100 \mathrm{~cm}=100 \mathrm{hPa}$ ).

4. Calculate the water content (WC) at $80 \%$ PAW: WC $\left(\mathrm{cm}^{3} \mathrm{~cm}^{-3}\right)=0.80(F C-P W P)+$ PWP. For the hydraulic limits of the soil used here this gives a volumetric water content at $80 \%$ PAW of $0.31 \mathrm{~cm}^{3} \mathrm{~cm}^{-3}$.

5. Calculate the water amount for a rhizobox volume of $2850 \mathrm{~cm}^{3}(30 \mathrm{~cm}$ width, $1 \mathrm{~cm}$ inner space, $95 \mathrm{~cm}$ in height, with top $5 \mathrm{~cm}$ keeping free of substrate for watering). This gives a water volume of $883.5 \mathrm{~cm}^{3}$ equal $883.5 \mathrm{~g}$ for the density of water being $1.0 \mathrm{~g}$ $\mathrm{cm}^{-3}$ at $20^{\circ} \mathrm{C}$.

3. Define the bulk density $\left(d_{b}\right)$ to fill the rhizoboxes. Here, set at $1.3 \mathrm{~g} \mathrm{~cm}^{-3}$ corresponding to values typically found in agricultural field soils. The amount of dry substrate required to fill a rhizobox volume of $2850 \mathrm{~cm}^{3}$ at this $\mathrm{d}_{\mathrm{b}}$ equals $3705 \mathrm{~g}$ of dry soil.

4. Pre-wet the dry soil to a gravimetric water content of $0.108 \mathrm{~g} \mathrm{~g}^{-1}$ (equal to a volumetric water content of $0.14 \mathrm{~cm}^{3} \mathrm{~cm}^{-3}$ ) by adding $400 \mathrm{~g}$ of water for $3705 \mathrm{~g}$ of dry soil and mix it gently to obtain a homogeneous water distribution. Manually disrupt larger aggregates to keep the particle size $\leq 2 \mathrm{~mm}$.

5. Fill the pre-wetted soil into the opened rhizoboxes and compact it gently using a polystyrene sheet $(30 \times 10 \times 1.5 \mathrm{~cm})$ to cover the inner volume of the box, thereby resulting in a homogeneous $d_{b}$ of $1.3 \mathrm{~g} \mathrm{~cm}^{-3}$.

6. Add the remaining amount of water $(483.2 \mathrm{~g})$ to achieve the target water content of $0.31 \mathrm{~cm}^{3} \mathrm{~cm}^{-3}$ by spraying onto the surface with a spray bottle. Ensure small drop size to avoid surface structure degradation and homogeneous wetting. Keep the box on a balance during spraying to monitor the amount of water actually added to substrate.

7. Let the water redistribute for 10 minutes and then press the glass onto the surface and fix it with the side metal rails. The average final weight of rhizoboxes with wetted substrate was $17818 \pm 68 \mathrm{~g}(13230 \mathrm{~g}$ rhizobox weight $+3705 \mathrm{~g}$ dry soil $+883 \mathrm{~g}$ water $)$.

NOTE: The homogeneous water content filled in the horizontal boxes will redistribute when the boxes are set in their final position according to the resulting potential gradient. This is a physical process in all plant growth pots according to their geometry (height) and experimenters should be conscious on their pot hydraulics ${ }^{26}$.

\section{Climate room setup}

1. Equip the climate room (Figure 3) with 8 LED lamps providing homogeneous illumination of $450 \mu \mathrm{mol} \mathrm{m} \mathrm{m}^{-2}$ with spectral peaks at 440 (blue) and 660 (red) nm for optimum plant growth. 

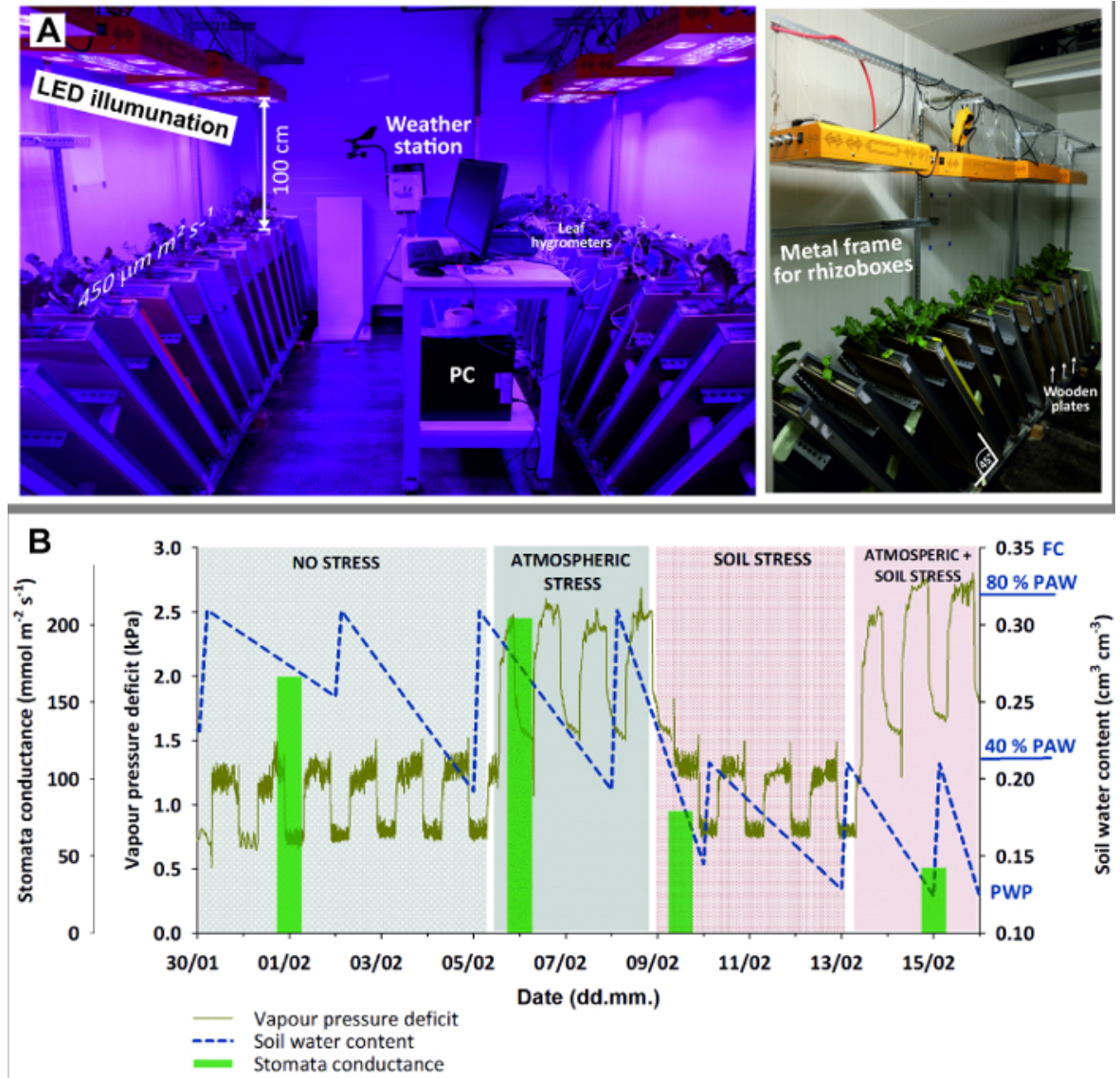

Figure 3: Climate chamber with rhizoboxes for stress experiment.

(A) Left view of entire chamber with LED illumination, weather station and PC (here for logging leaf hygrometers); right view with a closeup of the metal frame holding rhizoboxes in $45^{\circ}$ inclination and wooden plates used to shied rhizobox glass window against light. (B) Stress experiment with sugar beet combining four stages with stress due to different atmospheric demand (high/low) and soil water availability (high/ low). Green bars of mean stomata conductance give an indication of plant stress response. Please click here to view a larger version of this figure.

2. Set the ambient parameters according to plant/experimental needs. Here, use 14 hours light and 10 hours dark for illumination. During plant establishment and before stress treatments started, set the temperature to $20^{\circ} \mathrm{C}$ during day and $15^{\circ} \mathrm{C}$ during night and keep relative humidity at $50 \pm 8 \%$.

3. Put the rhizoboxes at an inclination of $45^{\circ}$ using an adequate metal framework. This maximizes root growth towards the glass surface due to gravitropism.

4. Cover the glass window by a wooden plate to keep the root zone in dark and avoid algae growth due to light penetrating through the glass surface.

\section{Sugar beet example setup and treatments}

1. Pre-germinate sugar beet seeds on wet filter paper for three days at $20^{\circ} \mathrm{C}$ in an incubator until the radicle emerges. This ensures seeding with viable plants.

NOTE: Pre-germination is not required for plants with high germination vigor, thereby avoiding the risk of damaging the radicle at seeding. For sugar beet seeds with a thick pericarp, however the risk of non-viable seeds is high and pre-germination significantly accelerates the emergence of the radicle compared to direct seeding into soil.

2. Drill a small hole to about $1.5 \mathrm{~cm}$ soil depth in the middle of the rhizobox with a screwdriver, position one seed in it using tweezers with the radicle oriented downwards and next to the glass window (this improves the initial visibility) and gently cover it with soil.

3. Add a $0.5 \mathrm{~cm}$ layer of fine gravel $(2-4 \mathrm{~mm})$ on top of soil to protect the soil aggregates from slaking during irrigation and reduce evaporation losses. To facilitate emergence, keep the soil surface free of gravel where the seed has been positioned.

4. Add $10 \mathrm{~g}$ of water to enhance establishment.

5. During establishment and early growth until experimental stress treatments start, irrigate the rhizoboxes every $2-4$ days to keep the initial moisture content of $80 \%$ PAW.

1. Determine the amount of irrigation water required by weighing the rhizoboxes and adding water until achieving the initial weight of each individual box. For manual irrigation use a pipette to avoid surface structure degradation during watering.

6. Arrange the rhizoboxes according to the established design in the climate room. The protocol reported here is based on an experiment with six sugar beet cultivars in five replicates in a completely randomized design (CRD). 
1. Reposition rhizoboxes each time when taking them out of the metal holder for weighing and watering. This avoids any effects of residual (light) inhomogeneity inside the climate room.

7. Define the time for onset of stress treatments and the type of stress. The following settings (Figure 4) are used here.

1. Start measurements at $\mathrm{BBCH} 15$ (five leaves unfolded) with roots covering around $75 \%$ of the rhizobox depth and the canopy being sufficiently developed for measurements at the leaves. Keep each stage for at least three days to ensure adaptation to the new settings and make measurement.

2. For the non-stress observations keep the initial settings with optimum soil moisture $(80 \% \mathrm{PAW})$ and ambient conditions $\left(20^{\circ} \mathrm{C} / 15^{\circ} \mathrm{C}\right.$ temperature, $50 \pm 8 \% \mathrm{rH}$ ) giving a daytime vapor pressure deficit (VPD) of $1.28 \pm 0.1 \mathrm{kPa}$ (cf. Figure $3 \mathrm{C}$ ).

3. Raise the atmospheric demand to a daytime VPD of $2.45 \pm 0.4 \mathrm{kPa}$ by increasing the temperature to $27^{\circ} \mathrm{C} / 20^{\circ} \mathrm{C}$ and decreasing $\mathrm{rH}$ to $35 \%$, while keeping soil moisture at $80 \%$ PAW.

4. Subsequently dry down the rhizoboxes to $40 \%$ PAW equivalent to a water content of $0.215 \mathrm{~cm}^{3} \mathrm{~cm}^{-3}$ by withholding irrigation. Reset the initial ambient conditions with low atmospheric demand (VPD of $1.28 \mathrm{kPa}$ ).

5. Combine stresses increasing the atmospheric demand to a VPD of $2.45 \mathrm{kPa}$ and keep soil moisture at $40 \%$ PAW.

\section{Root imaging methods}

1. Combine imaging methods to make use of their respective advantages and depending on the information targeted

1. Apply RGB imaging in the VIS range to track root growth, architecture and morphology over time which implicitly requires frequent measurement. Advantages of RGB imaging are (i) low costs, (ii) rapid image acquisition, (iii) low requirements of hard-disk space (image size: $48 \mathrm{MB}$ ) and (iv) high resolution (3648 $\times 5472$ pixels).

2. Use hyperspectral imaging (HSI) in the NIR range when chemometric features of root and soil are required. Advantages are (i) spectral features for segmentation between roots and soil background and (ii) access to physico-chemical system properties (e.g. soil water content, root age). Disadvantages are (i) higher scanning time (about 16 minutes per rhizobox), (ii) large size of datasets (13.7 GB per rhizobox image) and (iii) lower resolution of the NIR camera (320 x 256 Pixel), and (iv) higher complexity of data analysis.

\section{RGB root imaging}

1. Use an imaging box (Figure 4) that shields from ambient light and fixes the camera position consisting of a metal frame with a width of $1 \mathrm{~m}$ and a height of $1 \mathrm{~m}$ with side walls lined with pressboards. At the front end, fix the camera at two positions at a distance of $80 \mathrm{~cm}$ from the rhizobox.

1. Attach a tapeline on the rhizobox frame with transparent adhesive tape and position the rhizobox in the holder of the imaging box.

2. Illuminate the rhizobox using four $24 \mathrm{~W}$ fluorescent light tubes attached at a distance of $80 \mathrm{~cm}$ from the rhizobox. Also, mount four $15 \mathrm{~W}$ UV tubes at $20 \mathrm{~cm}$ from the rhizobox as alternative illumination making use of root auto-fluorescence in case of low contrast between root and (bright colored) substrate background.

3. Take two images (top and bottom position) to cover the upper and the lower half of a rhizobox with an overlap of about $3 \mathrm{~cm}$.

2. Acquire RGB images with a digital single-lens reflex camera which is fixed by quick release plates on the respective positions of the imaging box.

1. Apply the following settings when using the fluorescent light tubes. Adapt these example settings for any changes in dimensions and illumination as well as the camera model.

1. Turn off autofocus and stabilizer on the camera objective. Set camera to manual mode.

2. Set ISO speed to 500 . Set shutter speed to 13. Set Aperture to 5.6 .

3. Turn off the mirror lock. Set the white balance to Auto White Balance.

2. Use the following settings for illumination with UV tubes:

1. Turn off autofocus and stabilizer on the camera objective. Set camera in manual mode.

2. Set ISO speed to 1000 . Set shutter speed to 13. Set Aperture to 5.6.

3. Turn off the mirror lock. Set the white balance to Fluorescence.

3. Merge the RGB images from top and bottom of the rhizobox into a single image a photo editor (e.g., Adobe Photoshop). Use the tapeline at the side frame of the rhizoboxes and control overlapping objects (root and soil features).

1. Copy the two separate images, each with pixels size of $3648 \times 5472$, into a new file of size $3648 \times 10944$ pixels and white background.

2. Reduce the layer opacity for one image to $60 \%$ and align overlapping parts of the images (tapeline, objects). Thereafter restore layer opacity to $100 \%$.

3. Based on the ruler on the image add two red lines of exactly $1 \mathrm{~cm}$ to the top of the image where no roots are present. These lines are later used at image analysis to scale the image to correct length dimensions.

4. Merge all layers and remove parts of the image outside the soil filled window with the cropping tool.

5. Save the image as tiff-file for further analysis.

6. In case of images with UV illumination, reduce colors to greyscale before saving using the toolbar Adjustments-Black \& White, and selecting the predefined High Contrast Blue filter.

4. For segmentation between roots and soil background as well as for quantification of root traits of interest (e.g. length, surface, diameter, branching) use any root analysis software (see www.plant-image-analysis.org for available tools). Here WinRhizo is used.

1. Open the rhizobox tiff-image the software.

2. Calibrate the length scale of the image using the scaling bars added to the image.

3. Select Based on Color in the Analysis menu where selecting Root \& Background Distinction 
4. Define a calibration file with color classes corresponding to roots and soil (background). For the example images used here (cf. Figure 8) three root color classes (old laterals, young laterals, tap root) and three soil color classes are defined.

5. For the greyscale images (UV-illumination) select (i) Based on grey levels, and (ii) Pale Root on Black Background in the Root \& Background Distinction menu and use a local Lagarde intensity threshold for segmentation.

6. Open a data file where results are saved.

7. Run the analysis and subsequently control whether there are regions (e.g. at the edges) which are mismatched. In this case define an exclusion region and restart the analysis. For roots not classified, add additional color classes and restart the analysis. For elements wrongly classified as roots, activate/increase the Debris \& Rough Edges filtering options.
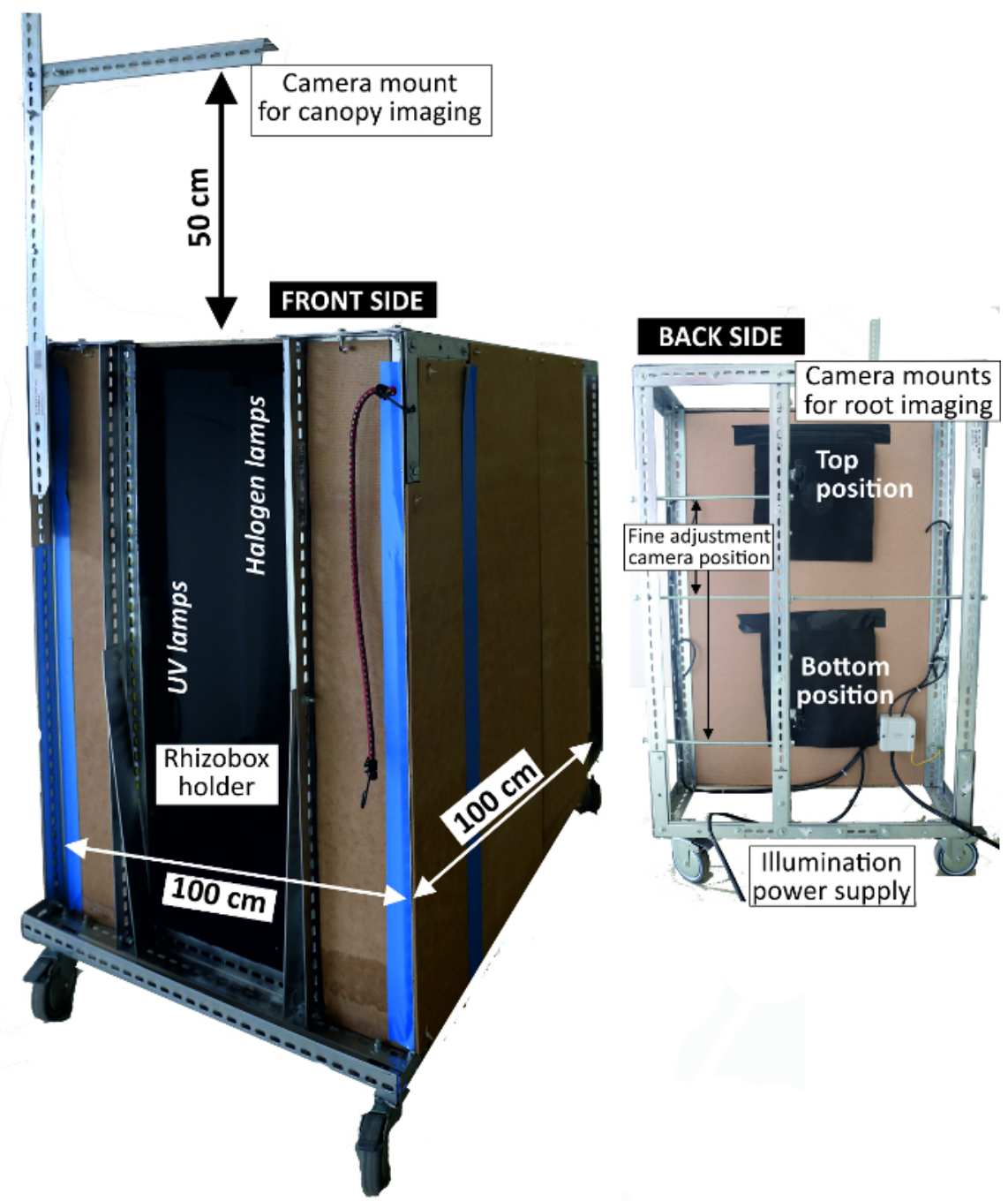

Figure 4: Imaging box to acquire RGB rhizoboxes pictures.

Left view of front side where rhizoboxes are attached for imaging with light sources inside; right view of backside where the camera is mounted. Please click here to view a larger version of this figure.

\section{Hyperspectral root imaging}

\section{Hardware setup}

1. Use a hyperspectral root imaging system (Figure 5) consisting of (i) a thermo-electrically cooled 14-bit monochrome NIR camera with a spectral range from $900 \mathrm{~nm}$ to $1700 \mathrm{~nm}, 320$ by 256 pixels and a frame rate of $100 \mathrm{~Hz}$ and (ii) an imaging spectrograph with a spectral range $900 \mathrm{~nm}$ to $2500 \mathrm{~nm}$ and a spectral resolution of $3.6 \mathrm{~nm}$. Arrange a halogen line illumination source (four 50 W halogen spots) in a $45^{\circ} /-45^{\circ}$ geometry. Mount the imaging sensor on a two-axis positioning system. The scan window has a size of $240 \times 1000 \mathrm{~mm}$, i.e. $30 \mathrm{~mm}$ at each edge of the rhizobox are not covered by the image.

2. Control the system by a Matlab script for (i) white and dark standard acquisition, (ii) setting of camera integration time, (iii) selecting spatial (pixel size $0.1 \mathrm{~mm}$; pixel size $1.0 \mathrm{~mm}$ ) and spectral resolution (all 222 spectral bands with a resolution of $3.6 \mathrm{~nm}$; smoothed spectrum with 54 bands and a resolution of $14.8 \mathrm{~nm}$ ), and (iv) defining the scan region on the rhizobox.

3. Save images as SIF-files. To avoid problems during saving of large files, subdivide each scanned image stride (9 strides per rhizobox) into four segments (three of $300 \mathrm{~mm}$ length, one of $100 \mathrm{~mm}$ length) and save separately with a unique file name consisting of stride number ( 1 to 9 ) and part (1 to 4 ) as well as date and time (YYYY.MM.DD HH:MM:SS). An entire rhizobox scan requires a hard-disk space of $13.7 \mathrm{~GB}$. 
2. Image acquisition and analysis.

NOTE: Figure 6 shows the steps of image acquisition, segmentation and analysis.

1. Image acquisition comprises selection of camera setting for optimum image quality and definition of scan parameters.

1. Determine the camera integration times for the rhizobox scan and the white standard in the camera software.

1. Open the imaging GUI and move the camera to a position of the rhizobox where roots are present.

2. Adjust the integration time of the camera targeting a light object (i.e. root) in a way that approximately $85 \%$ of the full dynamic range of the camera is used on the histogram displayed by the software. Repeat for the white standard by moving the camera positioning system to target the white standard. Then close the camera software.

2. Open the Matlab Imaging GUI and make all settings for the current rhizobox scan. For the data reported here, use the following settings:

Integration time white standard: 1000

Integration time rhizobox: 4000

Spectral resolution: Full resolution (i.e. 222 narrow-range spectral bands)

Full spatial resolution (pixel size of $0.1 \mathrm{~mm}$ )

1. Acquire the dark and white standards before each imaging run, e.g. once a day. The dark standard represents the camera noise, while the white standard gives the maximum reflectivity. These data are required for image normalization during pre-processing.

2. Define whether the entire rhizobox or only part of it is scanned. For the present case entire rhizoboxes are imaged. Then start the scan.

2. Process the image with a Matlab script. Operations performed by the script are described.

NOTE: Scripts are currently in an undocumented version and can be obtained from the corresponding author. After proper documentation, they will be available for download from the website of the corresponding author's institution (www.dnw.boku.ac.at/pb/).

1. Compose an entire image stride from the rhizobox center (containing roots) merging the four parts of the stride. NOTE: At this stage it is neither necessary nor recommended to use a spectral image of an entire rhizobox (i.e. all 9 strides) as the file size will make each calculation step in Matlab very time consuming and the information contained in one central stride is sufficient for the first steps of image analysis.

2. Normalize the image using the acquired dark and white standards and taking into account the different integration times of white standard and rhizobox scans which are saved automatically during scanning in a file.

3. Optionally apply a smoothing filter to remove noise from the image. The script currently offers $3 \times 3$ kernel median filtering and multiple scatter correction. For the image evaluation presented here, no filters are applied.

4. Display the image at all recorded spectral bands to obtain a first insight and decide a wavelength to be displaying for selecting regions of interest ( $c f$. image segmentation).

3. Perform segmentation between roots and soil background in a separate script with the following steps.

1. Select regions of interest (ROI) for root and soil to find spectral features for segmentation. Use the freehand selection tool to mark a ROI on the image displayed at a wavelength previously identified with good contrast between roots and soil. Here, use three ROIs on the root (old and young laterals, tap root) and two ROIs in the soil (dry, wet region).

2. Display a rectangle with the selected ROIs and the remaining part as a black mask and visualize the selected ROI image at all wavelengths.

3. Remove all lines in the image matrix containing pixels of spectral intensity $=0$ (black pixels).

4. Fuse the root and soil ROls into one foreground (root) and one background (soil) matrix for segmentation.

5. Search spectral bands (intensity of single spectra or spectral ratios) providing the best separation between root foreground and soil background ${ }^{27}$. Quantify the distinction between the resulting pixel histograms for root and soil using Bhattacharyya distance $^{28}$

6. Select a threshold intensity value separating the histograms.

7. Create a binary image by applying the selected threshold to the original image. This sets all pixels having smaller intensity than the threshold to zero and those with higher intensity to one (done automatically be the script).

8. Save the binary image as tiff-file.

4. Open the binary image and analyze root traits. Select (i) Based on grey levels, and (ii) Pale Root on Black Background in the Root \& Background Distinction menu and use global intensity threshold (the image is already binarized).

5. For mapping the water content from the hyperspectral data acquire a calibration dataset and apply a calibration equation to a rhizobox image.

1. Subdivide a rhizobox into $5 \mathrm{~cm}$ compartments using polystyrene sheets to fill them with soil (same substrate and $d_{b}$ as used for the experiment) at different water contents (Figure 8).

2. Calculate the respective amounts of water to be mixed with soil and fill the compartments (same procedure as described in 1.3. for the entire rhizobox).

3. Scan the calibration rhizobox with the same settings as used for the planted rhizoboxes.

4. Perform the following steps using a script.

1. Merge the four parts of a stride from the water calibration box to one stride and normalize it with dark and white standards.

2. Select rectangular boxes at each compartment with different water content and save them in a structure array.

3. Determine the spectral feature that best separates the water content compartments. This is done with a search algorithm for the global maximum of the intensity differences between mean spectra of adjacent water contents.

4. Calculate the mean spectral intensity value for this feature for each water content compartment of the calibration rhizobox. 
5. Fit a regression equation through the directly measured water content and the respective spectral quantifier.

6. Apply the regression equation to each pixel not classified as root on a rhizobox image and for the spectral feature (band) determined above which best relates to water content.

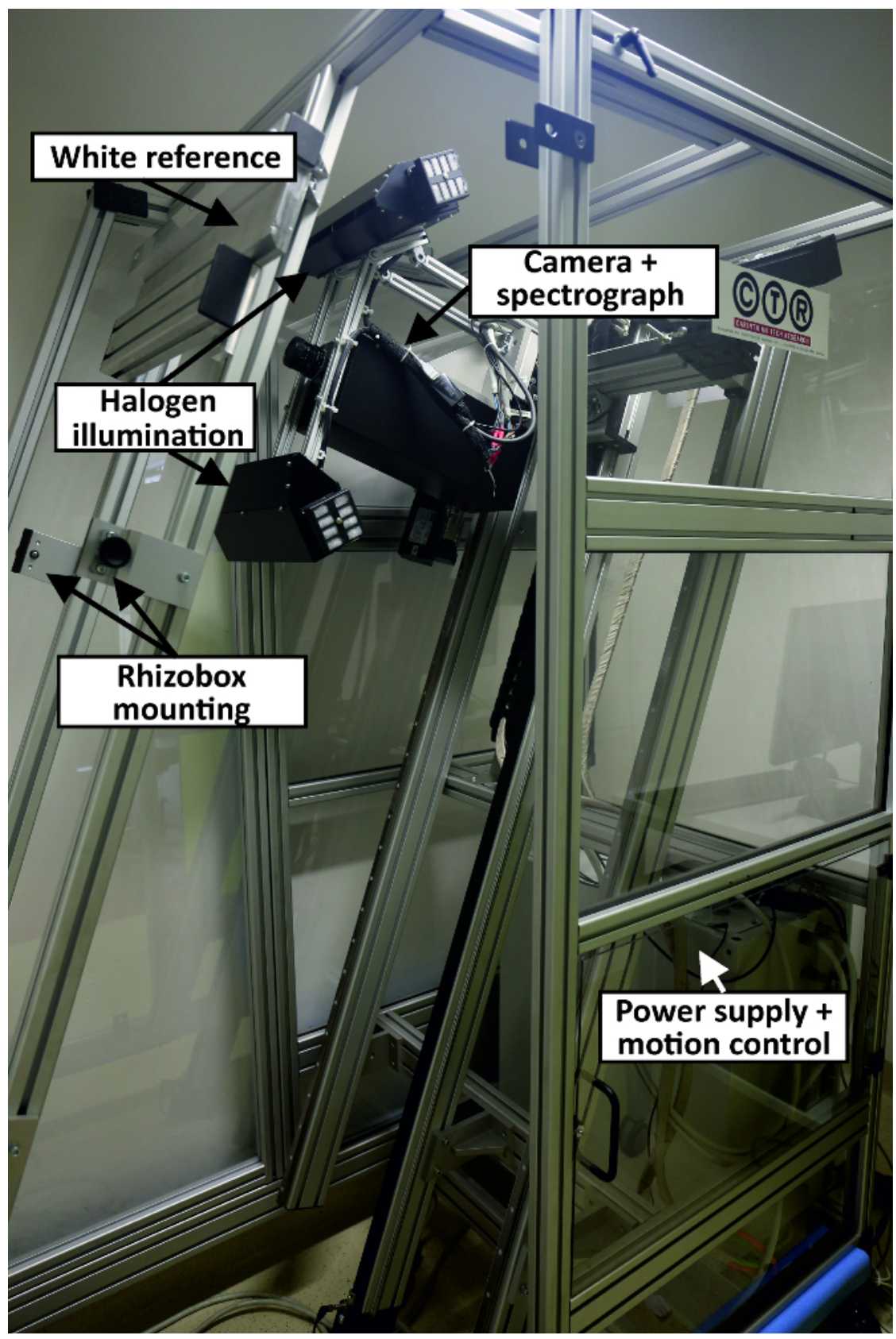

Figure 5: Hyperspectral root scanner.

The main components of the scanner are indicated. The small picture shows the camera during imaging of a rhizobox. Please click here to view a larger version of this figure. 


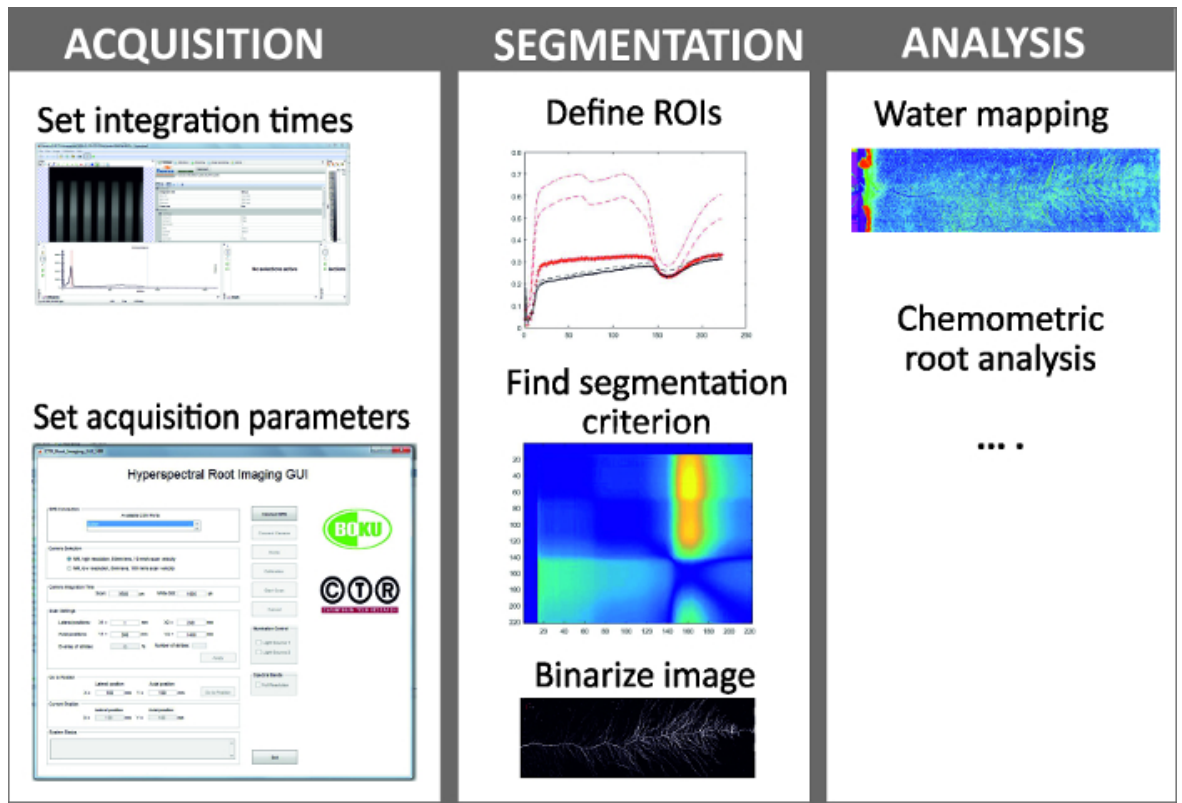

Figure 6: Steps in hyperspectral root imaging.

Hyperspectral root imaging consists of three mains steps being (i) image acquisition, (ii) image segmentation and (iii) analysis of the spectral data. Please click here to view a larger version of this figure. 


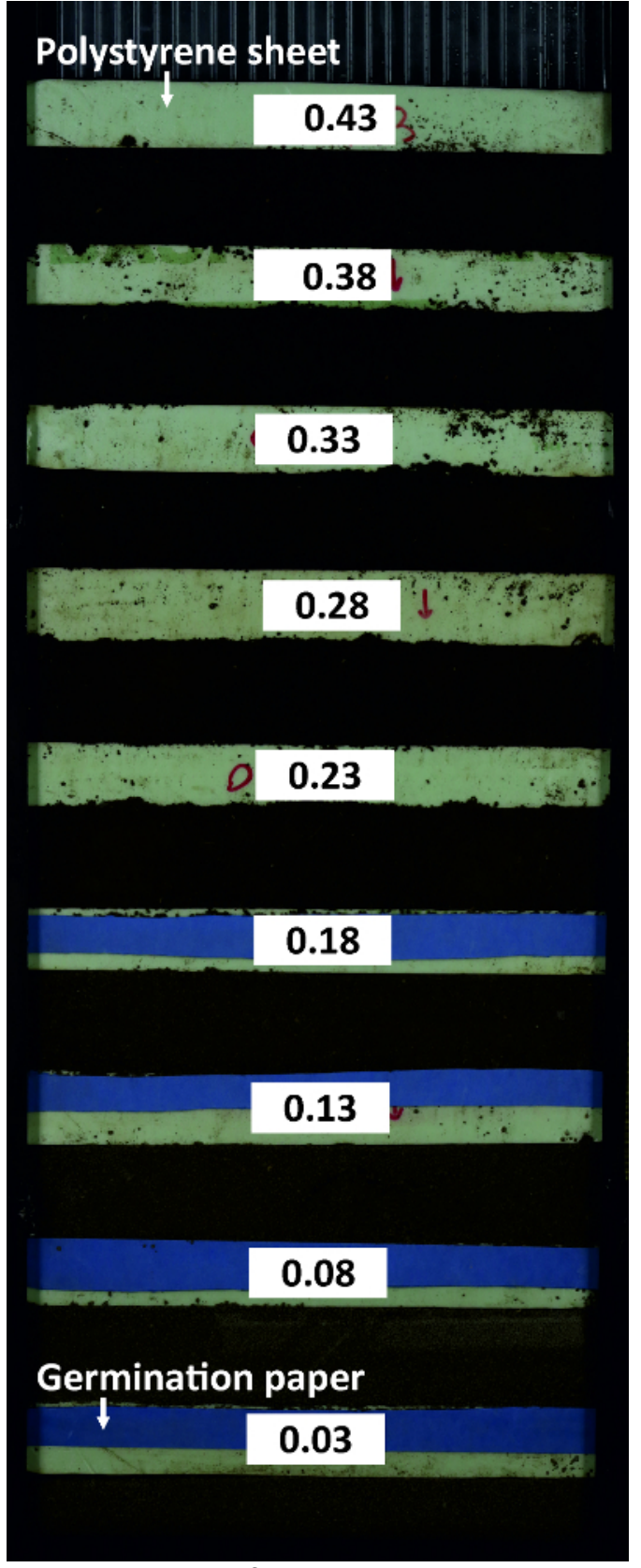

Figure 7: Rhizobox for water calibration.

The rhizobox contains compartments with substrate at different water content which are subdivided by polystyrene sheets. Germination paper at the dry compartments ensures that soil particles do not rinse into neighboring compartments. Please click here to view a larger version of this figure.

\section{Application examples}

NOTE: Quantitative root information is applied in the context of plant phenotyping (cultivar comparison) and for plant physiological research. The following aboveground data are reported to exemplify these cases.

1. Leaf area: Measure leaf area non-destructively at selected stages during the experiment via the length and width of leaves as a proxy. Alternatively canopy images can be used ${ }^{29}$.

1. At the end of the experiment measure leaf length and width together with area of clipped leaves using a leaf area meter. Calibrate the non-destructive method applying a regression equation to the data pairs.

2. Dry matter: At the end of the experiment, measure aboveground dry matter by clipping the plants with scissors and dry them for 24 hours at $105^{\circ} \mathrm{C}$ in an oven. 
3. Stomata conductance: Measure stomata conductance with a leaf porometer. Before measurement, keep the device for at least one hour in the climate chamber to allow sensors equilibrate with ambient conditions and calibrated the device each time when ambient settings in the climate chamber are changed. Take measurements from at least three leaves per plant.

\section{Representative Results}

Example results are presented for root segmentation based on RGB and HS imaging. For spectral imaging an example of high resolution water mapping is provided. Finally results are shown that demonstrate the scientific context where image based root data are applied.

\section{RGB based root measurement}

Figure 8 shows an RGB root image time series of sugar beet cultivar Ferrara. The images reveal some artefacts from inhomogeneous illumination of the rhizoboxes, with brighter areas along the left side and different brightness at the overlapping area between top and bottom images.

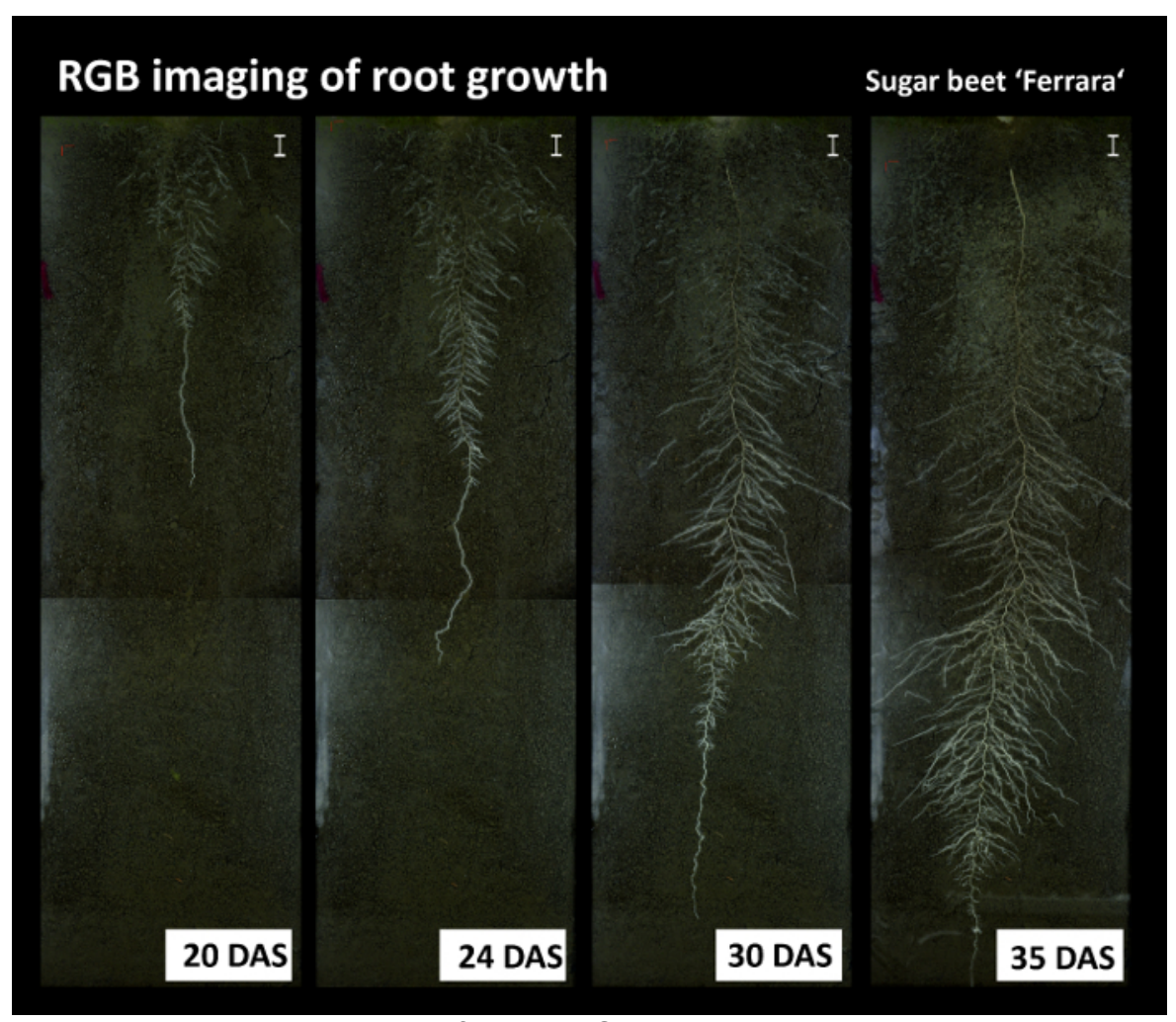

Figure 8: Root growth time series from the RGB imaging.

Pictures show the sugar beet cultivar Ferrara at different days after sowing (DAS). The images show some artefacts due to non-homogeneous illumination at the left side of the image and between top and bottom images. Scale bars, $2 \mathrm{~cm}$. Please click here to view a larger version of this figure.

Figure 9 provides details on root segmentation based on color thresholding for cultivar Ferrara at day after sowing (DAS) 35 . As a reference (Figure 9A), a binary image is used where all roots were manually tracked with a Graphic Tablet. The time required for manual tracking of the entire, fully developed, dense sugar beet root system was around four hours. Figure 9B gives a detailed view on a selected area at the top of the image where old lateral roots are present. Here several root axes are not classified by the color threshold. At the bottom (Figure $9 \mathrm{C}$ ) on the contrary, where white young roots are predominant, the color based segmentation properly classifies all root axes. The binarized root system (Figure 9D) shows a black area at the left side from the illumination artefact which was defined as exclusion region before running quantitative analysis. Figure $9 \mathrm{E}$ shows the corresponding pixel histograms of selected features (roots vs. soil) for the red channel of the RGB image from Ferrara at DAS 35. The root pixels (blue color) clearly show three peaks corresponding to bright young laterals, dark old laterals and tap root. The overlap between the old laterals and the soil background is very strong, leading to unclassified root axes (cf. Figure 9B). 


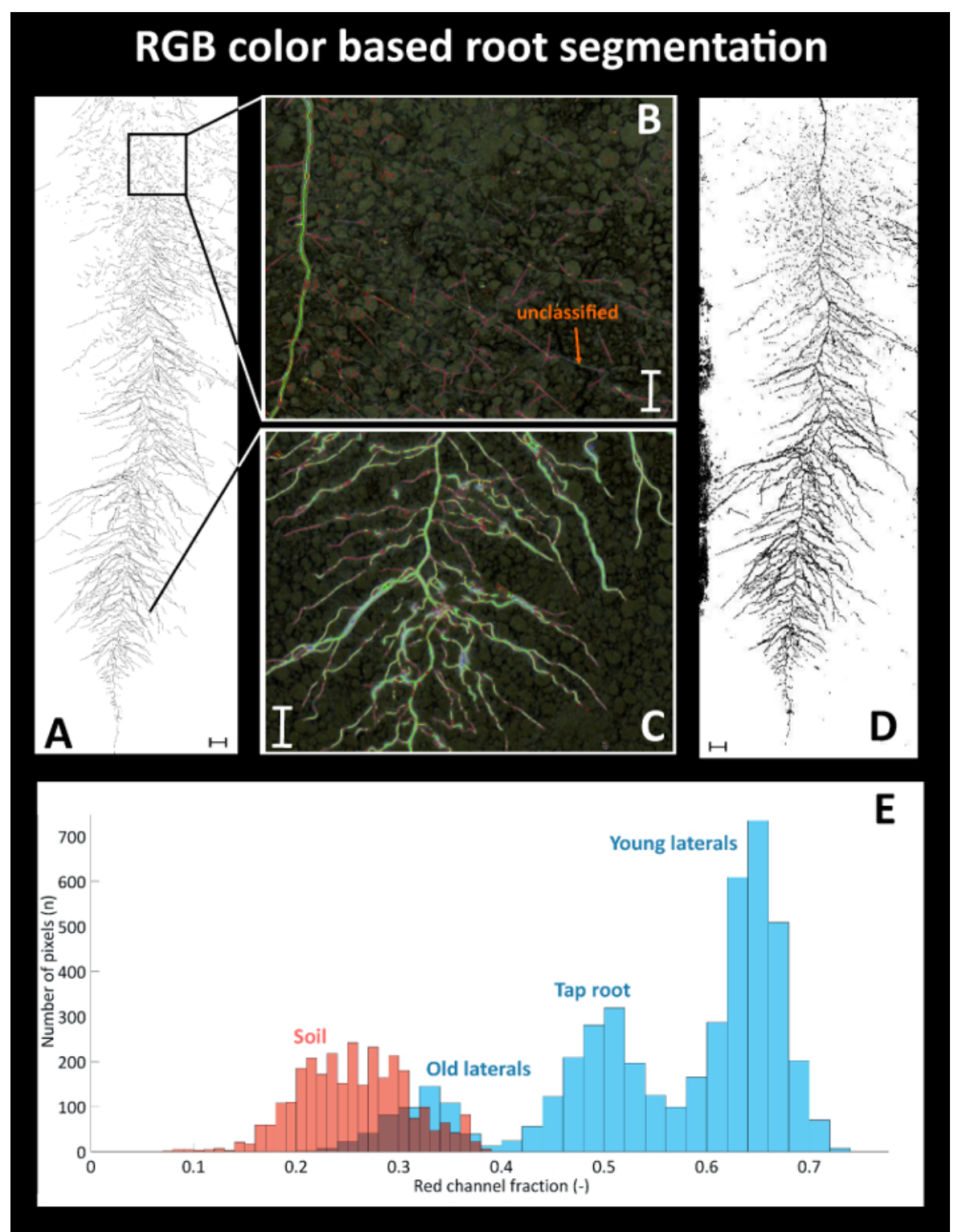

Figure 9: Root segmentation using a color threshold.

(A) Manually segmented root system using a Graphic Tablet, (B) area with poorly segmented old root axes in the top and (C) properly segmented young axes in the bottom of the image, and (D) binary image obtained from color based thresholding. (E) Pixel histograms for selected features of the RGB image. Roots are represented by the blue bars with different root types indicated; soil is represented by the red bars. Scale bars in A and D, $2 \mathrm{~cm}$; scale bars in B and C, $1 \mathrm{~cm}$. Please click here to view a larger version of this figure.

The resulting total visible root length quantified for the manually segmented reference image is $1534.1 \mathrm{~cm}$, while the automatized, color based segmentation gives a total root length of $1427.6 \mathrm{~cm}$.

Greyscale images from UV-illumination do not provide an advantage in the case shown here and performed worse compared to color thresholding (root length: $1679.7 \mathrm{~cm}$ ). Old roots could not be segmented, and there was more noise in the image, probably due to lower light intensity of the UV lamps. However, in case of young roots with high auto-fluorescence and a bright background substrate, UV-illumination can still be an option as shown by an image obtained from another experiment where sand was used as background substrate (Figure 10). 


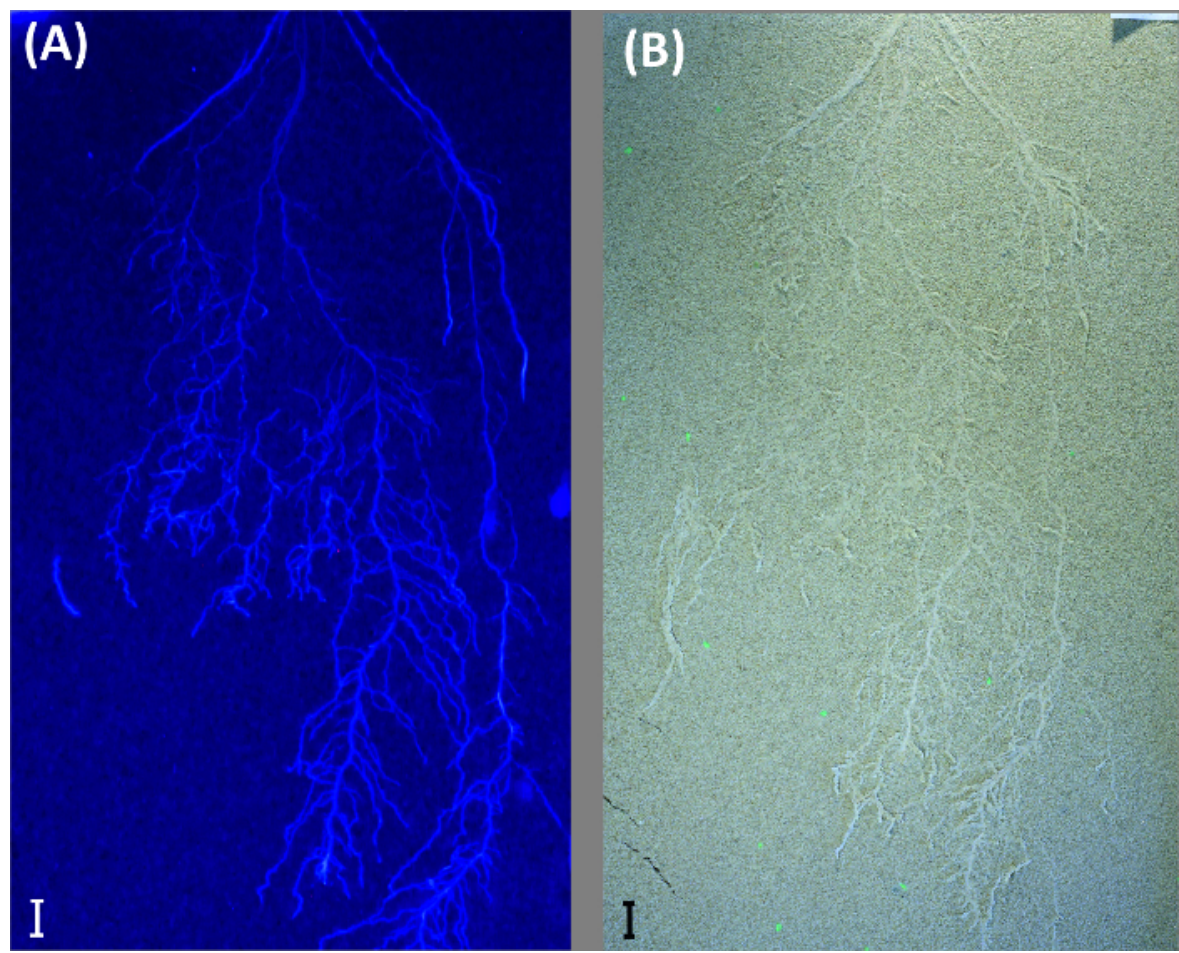

Figure 10: UV illumination to visualize roots on bright background.

Example from a durum wheat root system growing in a rhizobox filled with quartz sand. The rhizobox is imaged with illumination for (A) UV light and $(B)$ fluorescent (day) light. Scale bars, $2 \mathrm{~cm}$. Please click here to view a larger version of this figure.

\section{HSI based root measurements}

Figure 11 provides the mean spectra for three root ROls (old and young lateral, tap root) and two soil ROls (top and bottom of rhizobox).

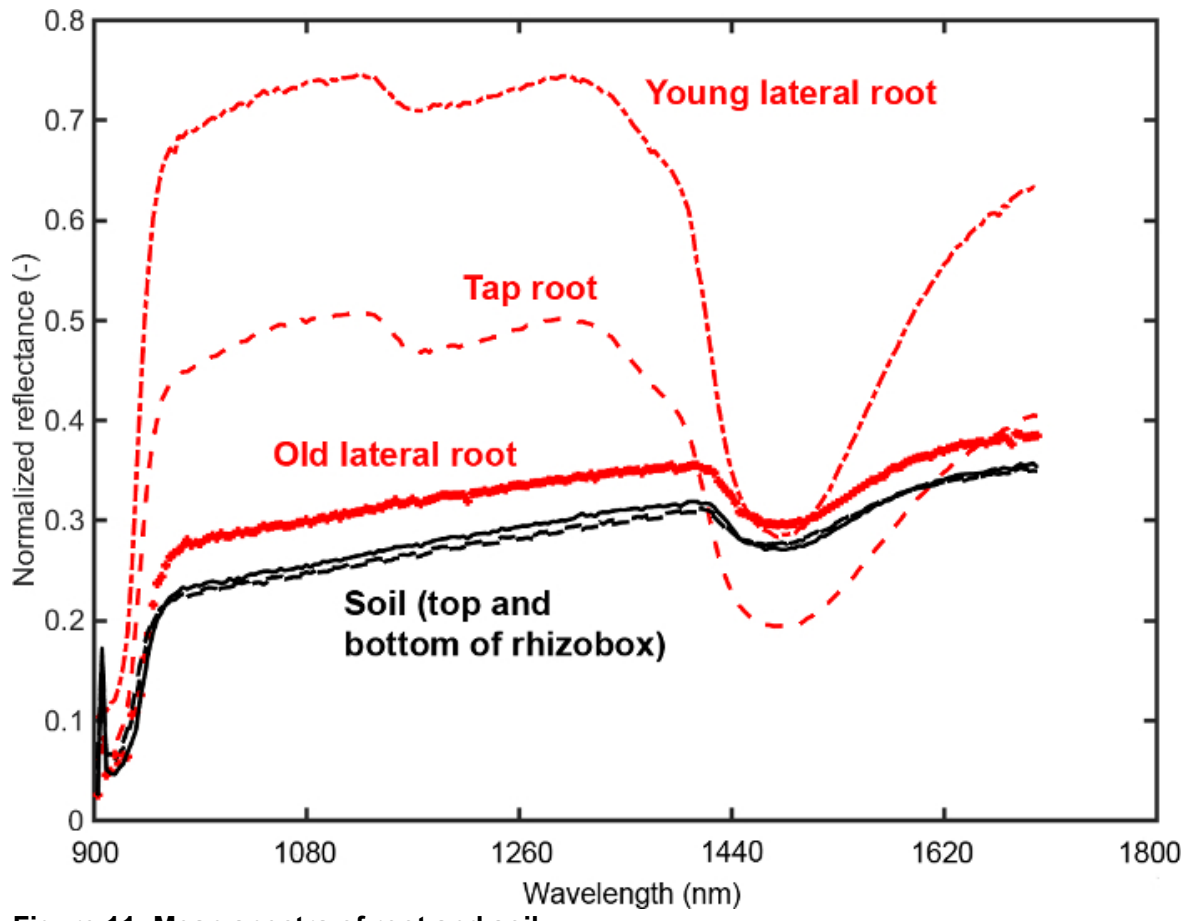

Figure 11: Mean spectra of root and soil.

Spectra from regions of interest (ROIs) on the root (three root types) and in the soil (top and bottom of the rhizobox). The ROIs are selected to determine an optimum segmentation criterion between root and soil. Please click here to view a larger version of this figure. 
It is evident that the tap and young lateral roots differ substantially from the background in intensity of most spectral bands. For the old laterals the intensity differences are much lower. A feature that can be inferred visually is the different slope of the spectrum around water absorption region $(1450 \mathrm{~nm})$. Here the slope of root spectra is higher compared to soil spectra. Furthermore a change of tap and young lateral spectra in the region around $1100 \mathrm{~nm}$ can be identified that does not occur in the old laterals.

Figure 12A shows the result from the search algorithm identifying a spectral ratio with strongest foreground-background contrast. The ratio of spectra at $1476 \mathrm{~nm}$ to $1076 \mathrm{~nm}$ provides the best separation between roots and soil. The resulting histogram of root foreground and soil background pixels is shown in Figure 12B. Although there is some overlap, most pixels are clearly separated from the soil background. Fitting a bimodal Gaussian curve through the histogram and using Bhattacharyya distance for quantification, a value of 7.80 is obtained. A value higher 3.0 indicates strong image contrast allowing reliable separation ${ }^{28}$.
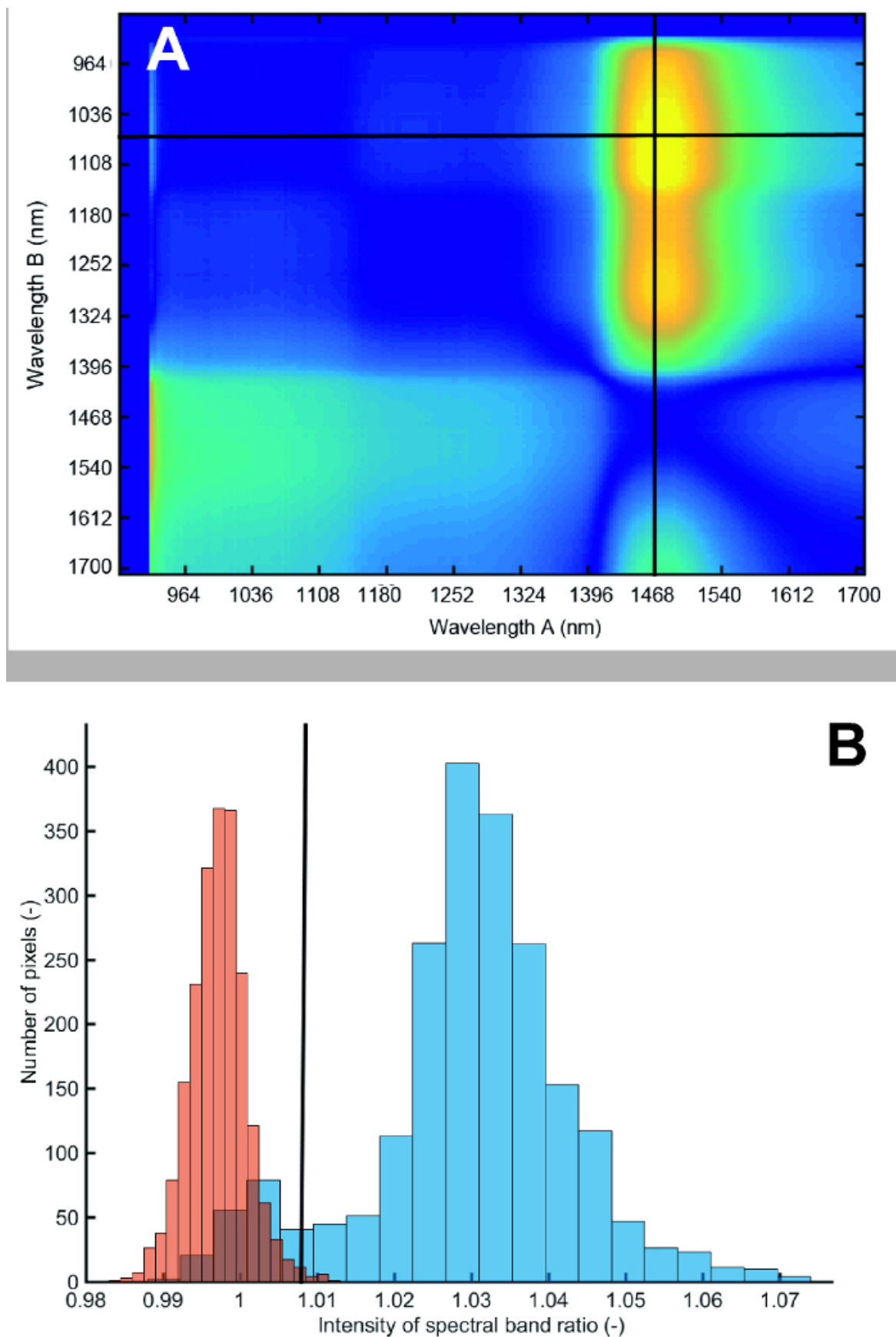

Figure 12: Difference in reflectance between root foreground and soil background for different spectral band ratios and pixel histogram at spectral ratio used for segmentation.

(A) Bright colors (yellow) show high contrast between foreground and background, dark colors (blue) show low contrast. The first 15 bands have been removed because of noise. The red lines indicate the band ratio with highest contrast. (B) Pixel histogram of roots (blue) and soil (red) at segmentation spectral ratio. Blue bars represent the root and red bars the soil. The intensity value corresponds to the ratio of spectral band 160 to spectral band 49. Please click here to view a larger version of this figure. 
The binary image (Figure 13) is created by applying a global intensity threshold of the identified spectral ratio at a value of 1.008 calculated from the histogram distance ${ }^{27}$. Analysis of root length of this image gives a total length of $1557.3 \mathrm{~cm}$ which represents an error of only $1.5 \%$ compared to the manually tracked reference image.

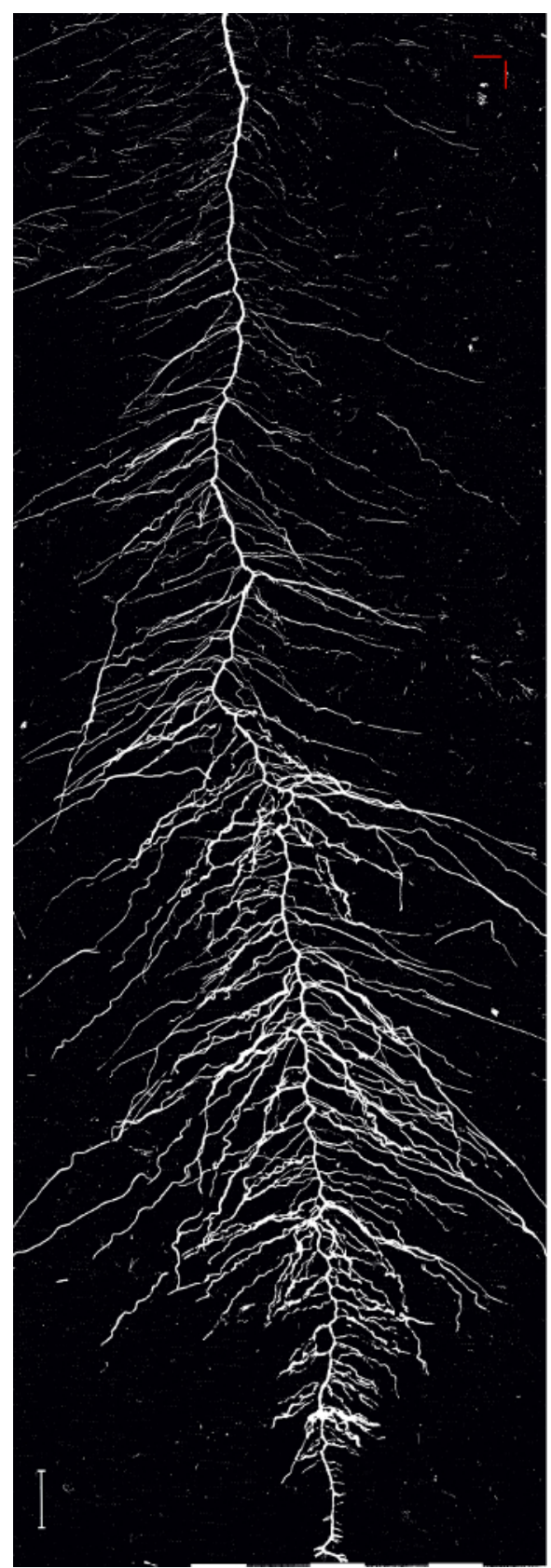

Figure 13: Binary image of the root system of sugar beet cultivar Ferrara.

The image is obtained by applying a global spectral threshold. Scale bar (bottom left corner), $2 \mathrm{~cm}$. Please click here to view a larger version of this figure.

Although root segmentation has improved using spectral information compared to color based information, the main intention of HS imaging is analysis of chemometric image properties. This is exemplified via mapping the water content of a rhizobox image.

Figure 14A shows the mean spectra of the compartments in the calibration rhizobox (cf. Figure 7) filled with soil of different water content. The shape of spectra is similar between the compartments, i.e. here a spectral ratio does not necessarily provide a more stable classification criterion. Thus intensity at a single spectral band $(1680 \mathrm{~nm})$, where the average difference between adjacent water contents is maximized, is identified as best separating criterion. The resulting pixel histograms for this spectral wavelength are shown in Figure 14B. 
(A)
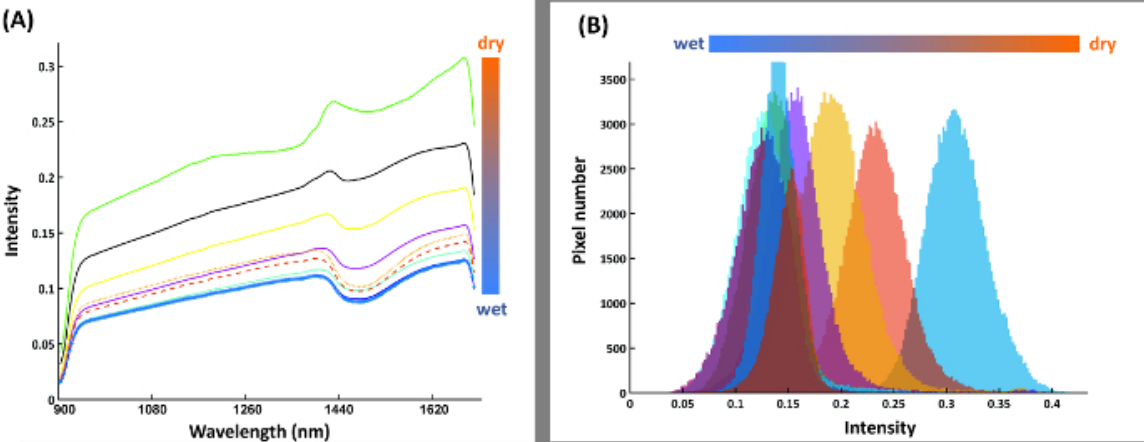

Figure 14: Spectral features for water content calibration.

(A) Mean spectra of nine water compartments from the calibration rhizobox with different water contents; (B) Pixel histograms for the water compartments at band 216 where average distance between neighboring compartment is maximum. Please click here to view a larger version of this figure.

The relation of the average pixel intensity at $1680 \mathrm{~nm}$ and the measured water content is shown in Figure 15.

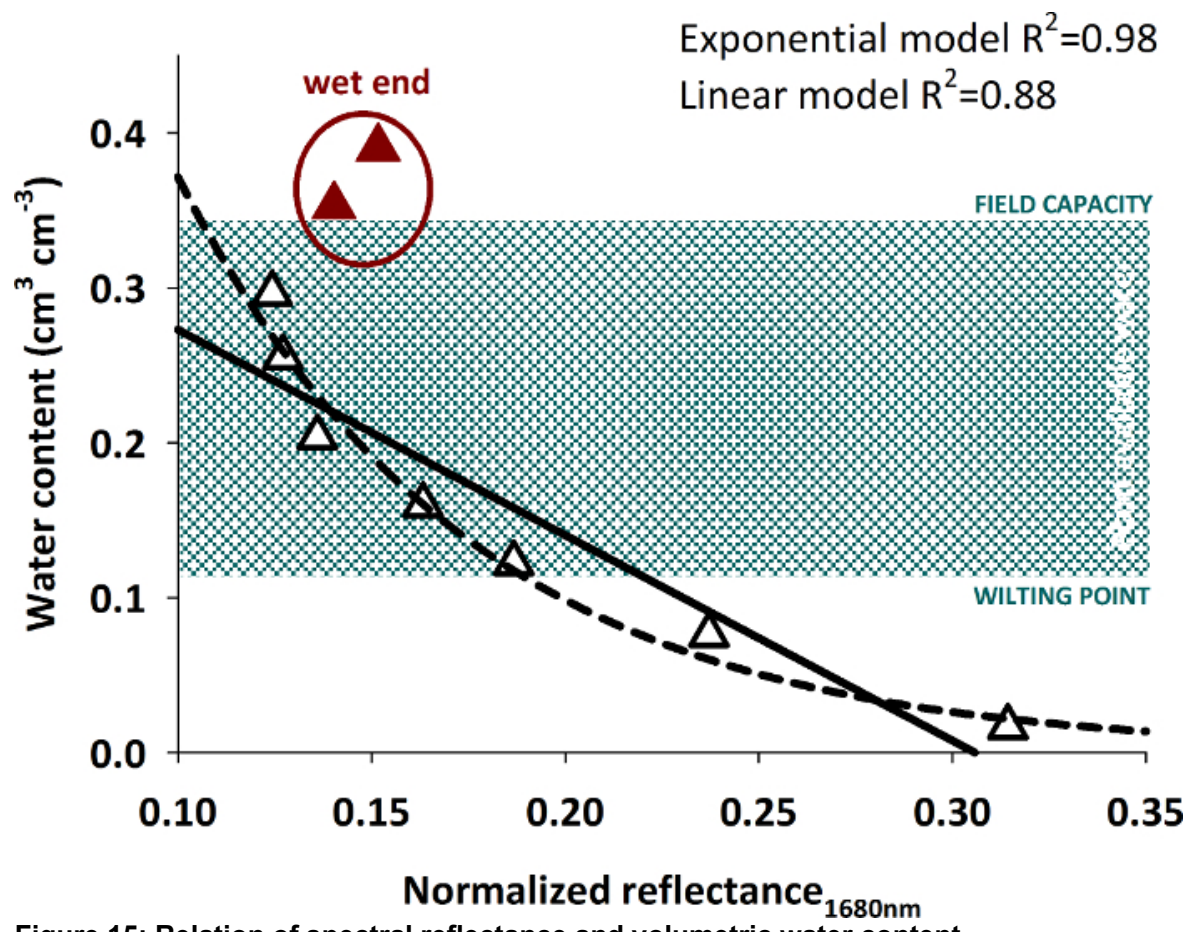

Figure 15: Relation of spectral reflectance and volumetric water content.

The figure shows data pairs of measured water content and spectral reflectance with empirical curves (linear and exponential) fit to the data excluding the highest water contents (red triangles). Please click here to view a larger version of this figure.

Differentiation of higher water contents from spectral intensity becomes difficult. A significant regression (either linear or exponential) with high $\mathrm{R}^{2}$ can be fit to water contents up to around $0.30 \mathrm{~cm}^{3} \mathrm{~cm}^{-3}$. Wetter soil conditions cannot be reliably predicted by the intensity value. Similar behavior of an exponential relation between reflectivity and water content with a decreasing response to water contents higher $0.30 \mathrm{~cm}^{3} \mathrm{~cm}^{-3}$ was also found in other studies ${ }^{30}$.

A rhizobox image with fine mapping of water content is shown in Figure 16. Four aspects have to be remarked. First, a region of lower water content can be seen in the rooted parts of the rhizobox. Second, strongest depletion is concentrated in the vicinity to single root axes. Third, depletion zones also occur where no root axes are visible on the surface, indicating regions where roots are hidden in soil. Fourth, water mapping without further image-processing results in a patchy appearance due to the aggregated soil. This can indicate inhomogeneous water content distribution at the aggregate scale, but also surface morphology effect on image quality. Chemometric image-processing techniques are an option to overcome such morphological effects in spectral images ${ }^{31}$, but are not implemented so far in the Matlab scripts used here. 


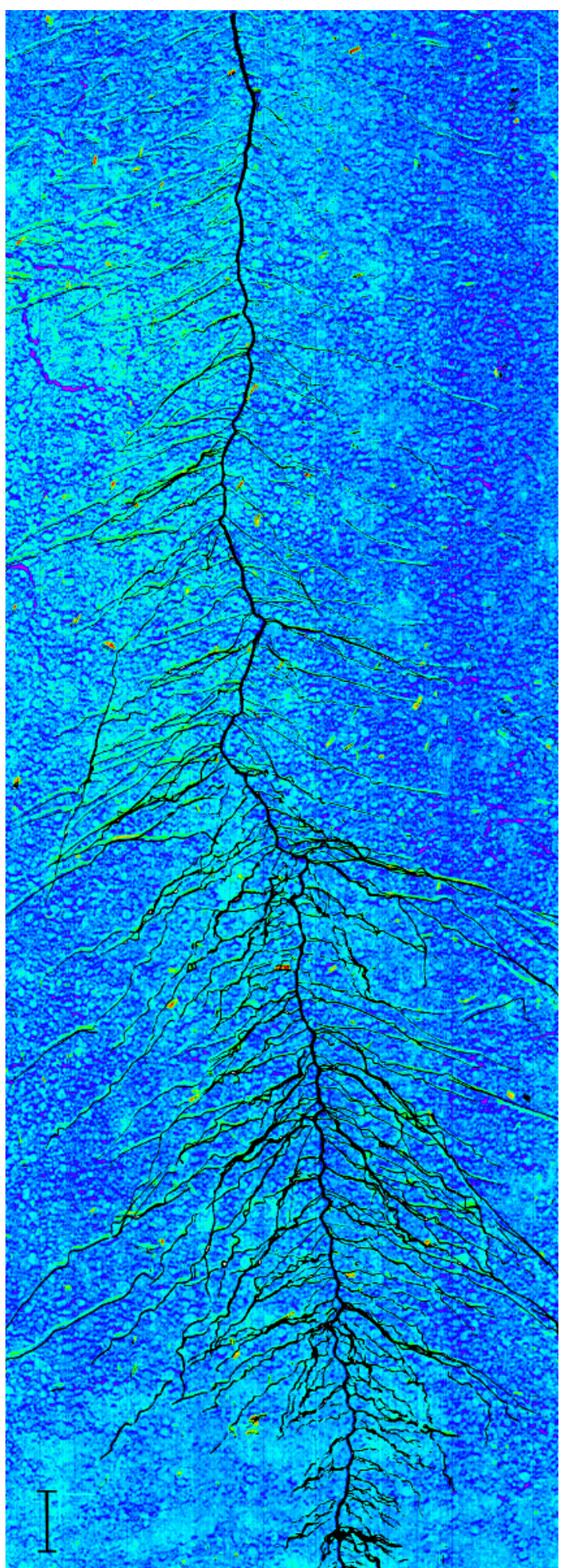

Figure 16: Water content mapping on a rhizobox.

The dark blue colours represent regions of high water content, green to red areas show regions with low water content. The plant root is overlaid on the image in black. Scale bar (bottom left corner), $2 \mathrm{~cm}$. Please click here to view a larger version of this figure.

\section{Application examples}

Figure 17 relates quantitative root traits from image analysis with aboveground measurements. 

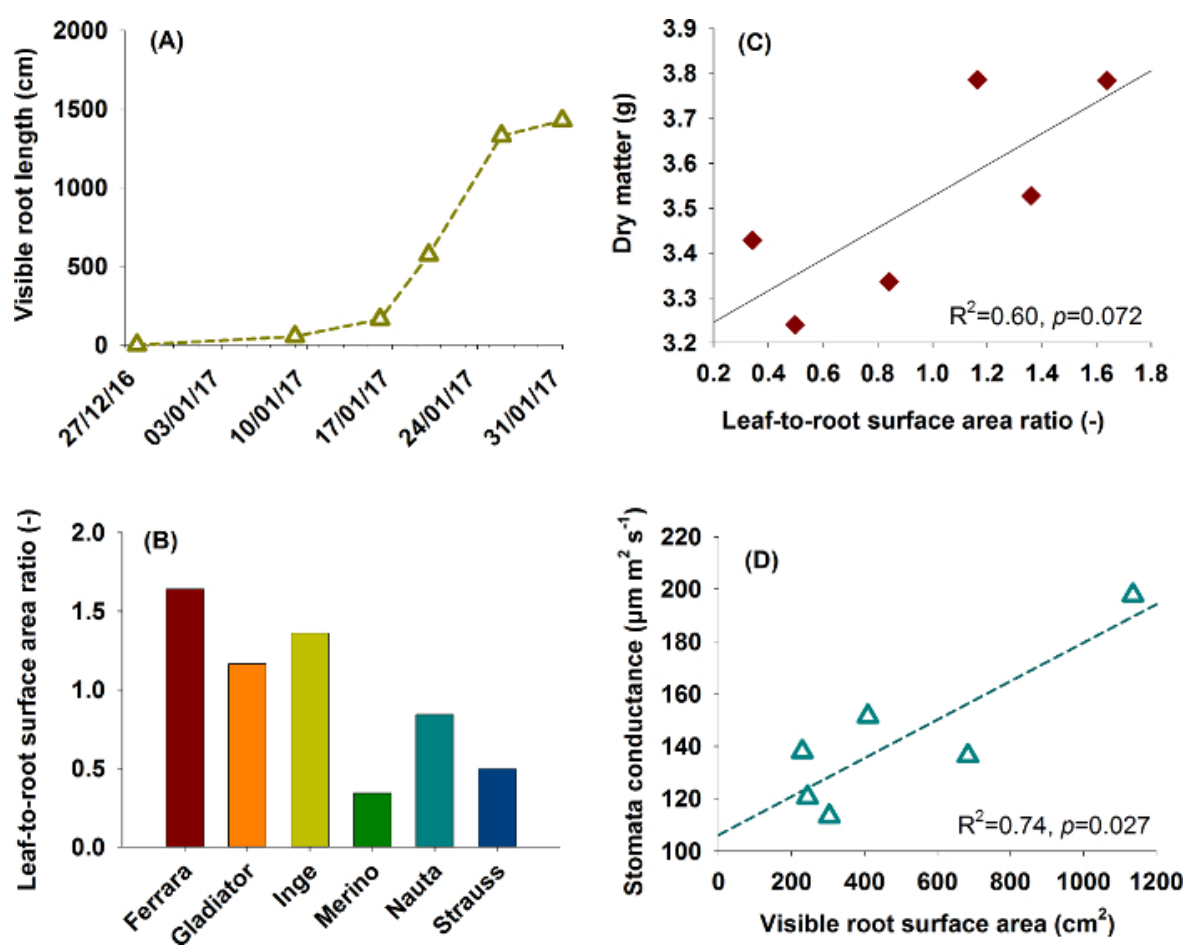

Figure 17: Typical application examples for root data.

(A) and (B) show root information used for aboveground-belowground plant characterization in a phenotyping context. (A) represents root growth from the sugar beet cultivar Ferrara, (B) compares six rhizobox grown sugar beet cultivars using leaf-to-root area ratio (data from one replicate).

(C) and (D) are functional relations between traits as found in plant physiological research. (C) shows the influence of leaf-to-root area ratio on dry matter production and (D) the relation of root surface area to stomata conductance. Please click here to view a larger version of this figure.

Figure 17A and 17B are relevant for phenotyping focusing on comprehensive aboveground and belowground plant characterization. Figure 17A shows root growth of sugar beet cultivar Ferrara (cf. Figure 8 for images). Expansion of the root system indicates the capacity of a cultivar to explore the soil volume in a given time span of the vegetation period. Figure 17B shows leaf-to-root surface area ratio of six sugar beet cultivars, providing a descriptor for the balance between plant supply (root) and demand (leaf).

Figures 17C and 17D give examples for functional relations of interest in physiological research. In Figure 17C leaf-to-root surface area ratio is related to dry matter formed during the experiment, indicating the predominant role of the assimilating surface as a limiting factor for dry matter accumulation. The lack of significance in spite of a comparatively high $R^{2}$ is related to the low number of paired data ( $\left.n=6\right)$ used here. Figure 17D reveals that cultivars with higher root surface area (improved uptake) have an average higher stomata conductance over the course of the experiment. The higher root area apparently sustains water extraction, thereby prolonging stomata opening.

\section{Discussion}

The protocols provide two complementary approaches for soil grown root system imaging. A critical step for reliable experimental results is filling of the rhizoboxes that has to ensure an even and homogeneous substrate layer at the front glass to provide tight root-soil contact at the observation window and avoid air gaps. This is the main reason to use comparatively fine sieved soil of < 2 mm: Larger aggregates result in higher surface morphology at the observation window with voids between aggregates. Besides a higher risk of root tip dehydration, this also requires more complex image processing techniques for water mapping ${ }^{31}$.

Modifications of the protocol therefore focus on improved and quick filling of rhizoboxes. Currently filling time is about 30 minutes per box. Furthermore use of rhizoboxes with two glass windows for imaging from both sides and modifications to optimize illumination homogeneity for better RGB images are tested. Further hardware extension might also consider integration of planar optodes ${ }^{32}$ as well as capacitance imaging $^{33}$ into the rhizobox system. This however is beyond current upgrading activities.

Software modifications focus on automatic image registration to fuse the top and bottom RBG images ${ }^{34}$. For hyperspectral imaging advanced unsupervised feature extraction approaches ${ }^{28}$ as well as more sensitive supervised target detection methods such as SVMs ${ }^{35}$ are tested. Thereby the hyperspectral data potentially allow for the assessment of multiple soil, rhizosphere and root properties ${ }^{36}$. Furthermore it is intended to develop a (semi)automatized software for rhizobox root images based on a modified version of Root System Analyzer ${ }^{37}$ to quantify morphological (length, diameter, surface) as well as architectural traits (branching frequency, branching angles).

The main limitation of the protocol compared to 3D imaging approaches is the restriction to the surface visible root and rhizosphere properties. However it has been demonstrated that the visible root traits are a reliable proxy for the whole root system ${ }^{21}$. The rhizobox technique is easily combined with traditional destructive sampling (washing) at the end of dynamic growth imaging in order to validate the relation of visible vs. total root system traits. As this relation might vary among species ${ }^{21}$, destructive sampling is recommended to ensure reliable inference from visible traits for any new phenotyping series with a different crop species. 
The key advantage of the protocol presented here is the combination of realistic growing conditions (soil), relatively high potential throughput for the temporally resolved RGB imaging and inference on root functionality (e.g. water uptake) via the chemometric root and rhizosphere data from hyperspectral imaging. Thereby the methods overcomes inference restrictions in high throughput seedling and non-soil root imaging methods ${ }^{14}$, while it partially allows deep phenotyping insights into functional processes with less experimental complexity and higher throughput compared to advanced $3 \mathrm{D}$ methods ${ }^{15}$.

In upcoming experiments the protocol will be used to study the effect of mycorrhiza on root system development and functionality of legumes as well as for phenotyping root characteristics of cover crop species in relation to soil structure, nitrogen and carbon cycling.

\section{Disclosures}

The authors have nothing to disclose.

\section{Acknowledgements}

The authors acknowledge funding from the Austrian Science Fund FWF via the Project Number P 25190-B16 (The roots of drought resistance). Establishment of the hyperspectral imaging infrastructure was supported financially by the Federal Government of Lower Austria (Land Niederösterreich) via the project K3-F-282/001-2012. Additional funding for the sugar beet experiment was received from AGRANA Research \& Innovation Center $\mathrm{GmbH}$ (ARIC). The authors thank Craig Jackson for technical support during the experiment and English correction of the manuscript. We also acknowledge Markus Freudhofmaier who contributed to establishment the RGB imaging setup and Josef Schodl for construction of the rhizobox mounting.

\section{References}

1. Kutschera, L. Wurzelatlas mitteleuropäischer Ackerunkräuter und Kulturpflanzen. DLG-Verlags-GmbH, Frankfurt am Main (1960).

2. Kenrick, P., \& Strullu-Derrien, C. The origin and early evolution of roots. Plant Physiol. 166, 570-580 (2014).

3. Franklin, J., Serra-Diaz, J.M., Syphard, A.D., \& Regan, H.M. Global change and terrestrial plant community dynamics. PNAS. 113, 3725-3734 (2016).

4. MacDonald, G.K., Bennett, E.M., Potter, P.A., \& Ramankutty, N. Agronomic phosphorus imbalances across the world's croplands. PNAS. 108, 3086-3091 (2011).

5. Vörösmarty, C.J., Green, P., Salisbury, J., \& Lammers, R.B. Global water resources: vulnerability from climate change and population growth. Science. 289, 284 (2000).

6. Lobell, D.B., Cassman, K.G., \& Field, C.B. Crop yield gaps: their importance, magnitudes, and causes. Annu. Rev. Environ. Resour. 34, 179-204 (2009).

7. Angus, J.F., \& Van Herwaarden, A.F. Increasing water use and water use efficiency in dryland wheat. Agron. J. 93, 290-298 (2001).

8. Lassaletta, L., Billen, G., Grizzetti, B., Anglade, J., \& Garnier, J. 50 year trends in nitrogen use efficiency of world cropping systems: the relationship between yield and nitrogen input to cropland. Environ. Res. Lett. 9(10), 105011 (2014).

9. Lynch, J.P. Roots of the second green revolution. Aust. J Bot. 55, 493-512 (2007).

10. Comas, L.H., Becker, S.R., Von Mark, V.C., Byrne, P.F., \& Dierig, D.A. Root traits contributing to plant productivity under drought. Front. Plant Sci. 4, 442 (2013).

11. Metcalfe, D.B. et al. A method for extracting plant roots from soil which facilitates rapid sample processing without compromising measurement accuracy. New Phytol. 174, 697-703 (2007).

12. Kutschera, L., Lichtenegger, E., \& Sobotik, M. Wurzelatlas der Kulturpflanzen gemäßigter Gebiete mit Arten des Feldgemüsebaues. DLGVerlag, Frankfurt am Main (2009).

13. Gioia, T. et al. GrowScreen-PaGe, a non-invasive, high-throughput phenotyping system based on germination paper to quantify crop phenotypic diversity and plasticity of root traits under varying nutrient supply. Funct. Plant Biol. 44, 76-93 (2017).

14. Watt, M. et al. A rapid, controlled-environment seedling root screen for wheat correlates well with rooting depths at vegetative, but not reproductive, stages at two field sites. Ann. Bot. 112, 447-455 (2013).

15. Fiorani, F., \& Schurr, U. Future scenarios for plant phenotyping. Annu. Rev Plant Biol. 64, 267-291 (2013).

16. Metzner, R. et al. Direct comparison of MRI and X-ray CT technologies for 3D imaging of root systems in soil: potential and challenges for root trait quantification. Plant Methods. 11, 1 (2015).

17. Choat, B., Badel, E., Burlett, R., Delzon, S., Cochard, H., \& Jansen, S. Non-invasive measurement of vulnerability to drought induced embolism by X-ray microtomography. Plant Physiol. 170, 273-282 (2015).

18. Metzner, R. et al. Direct comparison of MRI and X-ray CT technologies for $3 \mathrm{D}$ imaging of root systems in soil: potential and challenges for root trait quantification. Plant Methods. 11, 1 (2015).

19. Le Marié, C., Kirchgessner, N., Marschall, D., Walter, A., \& Hund, A. Rhizoslides: paper-based growth system for non-destructive, high throughput phenotyping of root development by means of image analysis. Plant Methods. 10, 1 (2014).

20. Bengough, A.G. et al. Gel observation chamber for rapid screening of root traits in cereal seedlings. Plant Soil . 262, 63-70 (2004).

21. Nagel, K.A. et al. GROWSCREEN-Rhizo is a novel phenotyping robot enabling simultaneous measurements of root and shoot growth for plants grown in soil-filled rhizotrons. Funct. Plant Biol. 39, 891-904 (2012).

22. Price, A.H. et al. Upland rice grown in soil-filled chambers and exposed to contrasting water-deficit regimes: I. Root distribution, water use and plant water status. Field Crops Res. 76, 11-24 (2002).

23. Vadez, V. Root hydraulics: the forgotten side of roots in drought adaptation. Field Crops Res. 165, 15-24 (2014).

24. Dane, J.H., \& Hopmans, J.W. Pressure plate extractor. In Dane, J.H., Topp, G.C. (Eds) Methods of soil analysis. Part 4. Physical methods. SSSA Inc., Madison, Wisconsin, USA, 688-690 (2002).

25. Wösten, J.H.M., Pachepsky, Y.A., \& Rawls, W.J. Pedotransfer functions: bridging the gap between available basic soil data and missing soil hydraulic characteristics. J. Hydrol. 251, 123-150 (2001). 
26. Passioura, J.B. The perils of pot experiments. Funct. Plant Biol. 33, 1075-1079 (2006).

27. Dorrepaal, R., Malegori, C., \& Gowen, A. Tutorial: Time series hyperspectral image analysis. J. Near Infrared Spec. 24, 89-107 (2016).

28. Kim, D.M. et al. Highly sensitive image-derived indices of water-stressed plants using hyperspectral imaging in SWIR and histogram analysis. Scie rep. 5, (2015).

29. Humplík, J.F., Lazár, D., Husičková, A., \& Spíchal, L. Automated phenotyping of plant shoots using imaging methods for analysis of plant stress responses-a review. Plant Methods. 11, 29 (2015).

30. Lobell, D.B., \& Asner, G.P. Moisture effects on soil reflectance. Soil Sci. Soc. Am. J. 66, 722-727 (2002).

31. Esquerre, C., Gowen, A.A., Burger, J., Downey, G., \& O'Donnell, C.P. Suppressing sample morphology effects in near infrared spectral imaging using chemometric data pre-treatments. Chemometr. Intell. Lab. 117, 129-137 (2012).

32. Williams, P.N., et al. Localized flux maxima of arsenic, lead, and iron around root apices in flooded lowland rice. Environ Sci. Technol. 48 , 8498-8506 (2014).

33. Cseresnyés, I., Takács, T., Végh, K.R., Anton, A., \& Rajkai, K. Electrical impedance and capacitance method: a new approach for detection of functional aspects of arbuscular mycorrhizal colonization in maize. Eur. J Soil Biol. 54, 25-31 (2013).

34. Brown, M., \& Lowe, D.G. Automatic panoramic image stitching using invariant features. Int. J. Comput. Vision. 74, 59-73 (2007)

35. Jiang, Y., Li. C., \& Takeda, F. Nondestructive detection and quantification of blueberry bruising using near-infrared (NIR) hyperspectral reflectance imaging. Scientific Reports. 6, 35679 (2016).

36. Chang, C.-W., Laird, D.A., Mausbach, M.J., \& Hurburgh, C.R. Near-infrared reflectance spectroscopy-principal components regression analyses of soil properties. Soil Sci. Soc. Am. J. 65, 480-490 (2001).

37. Leitner, D., Felderer, B., Vontobel, P., \& Schnepf, A. Recovering root system traits using image analysis exemplified by two-dimensional neutron radiography images of lupine. Plant Physiol. 164 (1), 24-35. (2014). 